\title{
Why I-Love-Q: Explaining why universality emerges in compact objects
}

\author{
Kent Yagi, ${ }^{1}$ Leo C. Stein, ${ }^{2}$ George Pappas, ${ }^{3}$ Nicolás Yunes, ${ }^{1}$ and Theocharis A. Apostolatos ${ }^{4}$ \\ ${ }^{1}$ Department of Physics, Montana State University, Bozeman, Montana 59717, USA \\ ${ }^{2}$ Center for Radiophysics and Space Research, Cornell University, Ithaca, New York 14853 USA \\ ${ }^{3}$ School of Mathematical Sciences, The University of Nottingham, University Park, \\ Nottingham NG7 2RD, United Kingdom \\ ${ }^{4}$ Department of Physics, Section of Astrophysics, Astronomy and Mechanics, University of Athens, \\ Panepistimiopolis Zografos GR15783, Athens, Greece \\ (Received 29 June 2014; published 23 September 2014)
}

\begin{abstract}
Black holes are said to have no hair because all of their multipole moments can be expressed in terms of just their mass, charge and spin angular momentum. The recent discovery of approximately equationof-state-independent relations among certain multipole moments in neutron stars suggests that they are also approximately bald. We here explore the yet unknown origin for this universality. First, we investigate which region of the neutron star's interior and of the equation of state is most responsible for the universality. We find that the universal relation between the moment of inertia and the quadrupole moment is dominated by the star's outer core, a shell of width 50\%-95\% of the total radius, which corresponds to the density range $10^{14}-10^{15} \mathrm{~g} / \mathrm{cm}^{3}$. In this range, realistic neutron star equations of state are not sufficiently similar to each other to explain the universality observed. Second, we study the impact on the universality of approximating stellar isodensity contours as self-similar ellipsoids. An analytical calculation in the nonrelativistic limit reveals that the shape of the ellipsoids per se does not affect the universal relations much, but relaxing the self-similarity assumption can completely destroy it. Third, we investigate the eccentricity profiles of rotating relativistic stars and find that the stellar eccentricity is roughly constant, with variations of roughly $20 \%-30 \%$ in the region that matters to the universal relations. Fourth, we repeat the above analysis for differentially rotating, noncompact, regular stars and find that this time the eccentricity is not constant, with variations that easily exceed $100 \%$, and moreover universality is lost. These findings suggest that universality arises as an emergent approximate symmetry: as one flows in the stellar-structure phase space from noncompact star region to the relativistic star region, the eccentricity variation inside stars decreases, leading to approximate self-similarity in their isodensity contours, which then leads to the universal behavior observed in their exterior multipole moments.
\end{abstract}

DOI: $10.1103 /$ PhysRevD.90.063010

PACS numbers: 97.60.Jd, 04.40.Dg

\section{INTRODUCTION}

Astrophysical observations of neutron stars (NSs) may reveal one of the most important "known unknowns" of nuclear physics: the relation between density and pressure, the so-called equation of state (EOS), of supranuclear matter [1-3]. One can constrain the EOS by measuring at least two NS observables that depend strongly on the star's internal structure. The most well-studied observables are the NS mass and radius (see e.g. [4] and references therein). Although the latter has not been measured to better than $20 \%$ accuracy, a Bayesian analysis has allowed some constraints on the EOS $[5,6]$ and on certain quantities in nuclear physics, such as the nuclear symmetry energy $[7,8]$.

Some relations among certain NS observables depend very weakly on the EOS. Such approximate universality has been found, for example, between the NS binding energy and compactness $[1,9,10]$, between the mass-shedding (Keplerian) frequency for rotating configurations and compactness for nonrotating configurations [11,12], among NS oscillation modes [13-16], among certain tidal parameters
[17], among gravitational-wave (GW) observables from NS binaries [18-23] and among the compactness, a dimensionless spin parameter and the effective gravitational acceleration on the surface of a rapidly rotating NSs [24].

A stronger, yet still approximate universality has been recently found among the moment of inertia $(I)$, the tidal and rotational Love numbers (or tidal deformability for the former) and the quadrupole moment $(Q)$ of NSs and quark stars (QSs) [25,26] (see also [1,27-30] for universal relations among $I, Q$ and the NS compactness). The relations were confirmed by [7] through a detailed study of different EOSs, by [31] for NS binary system that are strongly and dynamically tidally deformed, and by [32] for magnetized NSs, provided they are not slowly spinning magnetars. These relations also hold for rapidly rotating stars, as demonstrated numerically by [33-35] and analytically in [36,37], with the latter in the Newtonian limit.

The I-Love-Q relations have several important applications. First, a measurement of any one of these quantities automatically allows for the determination of the other two, 
without having to know the EOS. Second, these relations allow for model-independent and EOS-independent strong-field test of General Relativity (GR). For example, Refs. $[25,26]$ showed that measuring the NS Love number with GW observations and the moment of inertia from binary pulsars can place very stringent constraints on parity violations in gravity $[38,39]$. The relations were recently studied in $[40,41]$ for other classes of modified theories of gravity. Third, the I-Love-Q relations break degeneracies between certain parameters in astrophysical and GW observations. For example, the Love-Q relation can be used to break the degeneracy between $Q$ and spins in GW observations of spin-aligned binary NSs [25,26]. Moreover, the I-Q relation can be used to break degeneracies in X-ray pulse profile observations with NICER [42] and LOFT $[43,44]$, as shown in $[45,46]$.

The approximately universal I-Love-Q relations resemble the celebrated no-hair relations for black holes (BHs) [47-53]. Astrophysical (uncharged) BHs are said to have no hair because all of their multipole moments, i.e. the coefficients in a multipolar expansion of the gravitational field far from the source, can be written completely in terms of just their mass and spin angular momentum [47-53]. Ordinary stars or compact stars need not be bald, and thus, their multipole moments could depend strongly on the star's EOS.

Nonetheless, the approximate universal relations between $I$ and $Q$ and a new relation between $Q$ and the current octupole [33] for compact stars suggest the existence of an approximate no-hair relation. Recently, in fact, approximately universal no-hair relations were found for lower multipole moments $(\ell \leq 10)$ in terms of the first three (the mass monopole, the current dipole and the mass quadrupole), albeit in the nonrelativistic, Newtonian limit [36] and with a certain elliptical isodensity approximation [54] that models stellar isodensity contours as self-similar ellipsoids. Some of these results have been recently confirmed numerically and in full GR for the mass hexadecapole and the mass quadrupole moments [35] of compact stars.

But why does such approximate universality hold in the first place? When discovering the I-Love-Q relations, Refs. [25,26] suggested two nonexcluding hypothesis. One of them was that universality holds because all EOSs are somewhat similar in the NS region that dominates the I-Love-Q calculation, i.e. in the outer core. Indeed, our ignorance of the EOS is smaller toward the crust than toward the inner core of NSs. The other hypothesis was that universality holds because the I-Love-Q relations must approach the $\mathrm{BH}$ limit as the stellar compactness increases, and of course, for BHs these relations must be universal. These explanations are not quite satisfactory, however, because the EOS does vary in the outer core and NSs are not nearly as compact as $\mathrm{BHs}$; in fact, there is no continuous limit from a NS sequence to a $\mathrm{BH}$.

\section{A. Methodology and executive summary}

The purpose of this paper is to gain a better understanding of why approximate universal relations among multipole moments, in particular between $I$ and $Q$, hold for NSs. Since rotational Love numbers are directly related to $I$ and $Q$ [26], such reasoning will also explain the origin of the universal relation between the rotational Love number and $I$ or $Q$. We achieve this goal by investigating the universal relations in detail and addressing the following questions:

(i) Which part of the EOS and NS interior region is most responsible for the universality? Are the realistic EOSs similar to each other in this region?

(ii) Which assumption in the elliptical isodensity approximation is most important in the universality?

(iii) Does the elliptical isodensity approximation hold for realistic relativistic stars? What do the eccentricity radial profiles of such stars look like?

(iv) Does the elliptical isodensity approximation hold for realistic noncompact stars? Are there universal relations for such stars?

The first question is tackled as follows. We consider slowly rotating NSs and QSs in the Hartle-Thorne approximation $[55,56]$ with an extended piecewise polytropic EOS [57], characterized by five free parameters: $\left(p_{1}, \Gamma_{1}, \Gamma_{2}\right.$, $\left.\Gamma_{3}, \rho_{1}\right): p_{1}$ changes the overall normalization of the EOS, $\Gamma_{1}$ affects both the inner and outer core regions, $\Gamma_{2}$ and $\Gamma_{3}$ only modify the inner core region, and $\rho_{1}$ determines the transition density between the $\Gamma_{1}$ and $\Gamma_{2}$ regions. A version of such a piecewise polytropic EOS has been shown to recover realistic EOSs for suitable choices of the free parameters [57]. We vary $\left(p_{1}, \Gamma_{1}, \Gamma_{2}, \Gamma_{3}, \rho_{1}\right)$ from a fiducial set that recovers the realistic SLy EOS [58] to study how each parameter affects the universal I-Q relation, the radial profiles of the energy density and (the integrand of) the moment of inertia and quadrupole moment.

We find that, although all parameters have a large effect on the mass-radius relation, $p_{1}, \Gamma_{3}$ and $\rho_{1}$ hardly affect the relation at all, while $\Gamma_{1}$ and $\Gamma_{2}$ affect the I-Q relation the most for low- and high-compactness stars, respectively, and in a linear fashion. These results are confirmed by computing the radial profile of the integrand of the moment of inertia and quadrupole moment, which are most affected when we vary $\Gamma_{1}$ and $\Gamma_{2}$. We find that the moment of inertia and quadrupole moment are dominated by contributions within a shell inside the star of size $50 \%-95 \%$ of the total radius.

Figure 1 presents the radial profile of the energy density for nonrotating NS configurations with two different choices of dimensionless moment of inertia $\bar{I}=I / M^{3}$, where $M$ is the stellar mass. We vary each piecewise polytrope parameter by $30 \%$ from the corresponding value of the fiducial SLy EOS. The $90 \%$ contribution to the moment of inertia and the quadrupole moment comes from the radial region in vertical dashed lines. One sees that the 


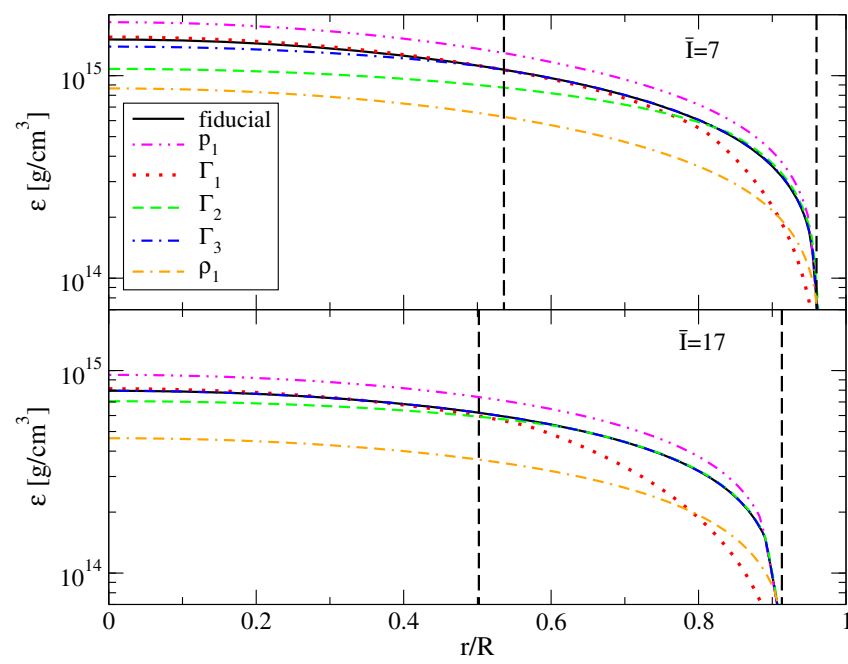

FIG. 1 (color online). Energy-density profile $\epsilon$ of nonrotating NSs with the dimensionless moment of inertia of $\bar{I}=7$ $\left(M \approx 1.8 M_{\odot}\right)$ (top) and $\bar{I}=17\left(M \approx 1.1 M_{\odot}\right)$ (bottom) for the fiducial SLy EOS, and with $30 \%$ variations of piecewise polytrope parameters $p_{1}, \Gamma_{1}, \Gamma_{2}, \Gamma_{3}$ and $\rho_{1} . R$ in the horizontal axis refers to the NS radius for nonrotating configurations. The region in between the vertical dashed lines corresponds to the one that contributes the most to the calculation of $I$ and $Q$. Observe that $\bar{I}$ is mostly affected by the EOS in the range $\epsilon=\left(10^{14}-10^{15}\right) \mathrm{g} / \mathrm{cm}^{3}$.

I-Q universality is mostly affected by the energy densities in the range $10^{14}-10^{15} \mathrm{~g} / \mathrm{cm}^{3}$. Although the NS EOSs all have a similar slope within such a region, they can differ by as much as $\sim 17 \%$, and hence this fact alone cannot explain the $\mathcal{O}(1) \%$ universality in the I-Q relation.

Since the region that matters the most for the universal relations is quite far from the NS inner core, we suspect that a Newtonian analysis might suffice to understand the reasons behind the universality. To determine if this is the case, we construct rapidly rotating NS solutions using the RNS code [59]. These solutions confirm that although relativistic and rotational effects make stars more centrallycondensed, the energy density profiles are not modified much in the region that matters. Thus, an analysis carried out in the nonrelativistic limit should be sufficient to understand why universality holds, which then brings us to the second question.

This question is tackled by studying the universal relations in the nonrelativistic, Newtonian limit, extending the work in [36] by relaxing the elliptical isodensity approximation. This approximation consists of three main conditions [54]: (i) that constant density contours are selfsimilar surfaces; (ii) that such surfaces are ellipsoids; and (iii) that the isodensity profile in terms of the isodensity radius is identical to that of a nonrotating star of the same volume. We relax each of these, one by one, and find that condition (ii) does not affect the universal relations at all, but conditions (i) and (iii) can destroy them. In particular, if one allows the stellar eccentricity to depend on the radial coordinate rather than being a constant, the EOS universality can be lost. This suggests that the self-similarity of the elliptical isodensity approximation plays an important role in the universality.

But are the isodensity contours of realistic NSs approximately self-similar? This is the focus of the third question we tackle, which we answer by constructing both slowly rotating and rapidly rotating NS solutions and extracting the stellar eccentricity in terms of the radial coordinate. Figure 2 shows the eccentricity profile for slowly rotating NSs with various EOSs. One sees that in the region that matters [i.e. $r \in(50 \%, 95 \%) R$, with $R$ the stellar radius], the eccentricity only changes by $\sim 10 \%$ for slowly rotating stars. For rapidly rotating NSs, we find that the eccentricity variation inside the star is always below $20 \%-30 \%$. We extend the Newtonian analysis described in the previous paragraph by using such realistic eccentricity profiles to correct the elliptical isodensity approximation. We find that this leads to changes of $\sim 10 \%$ at most relative to the universal relations obtained with constant eccentricity, e.g. if the $\bar{I}-\bar{Q}$ relations are universal to roughly $1 \%$, the variations in the eccentricity correct these relations to $0.1 \%$. This supports the validity of the approximation for realistic NSs.

Finally, we study whether noncompact stars are also approximately elliptically self-similar and whether the universal relations hold in this case. This is the basis of the fourth question described earlier, which we tackle by constructing realistic, differentially-rotating, noncompact stellar solutions with the publicly-available ESTER code

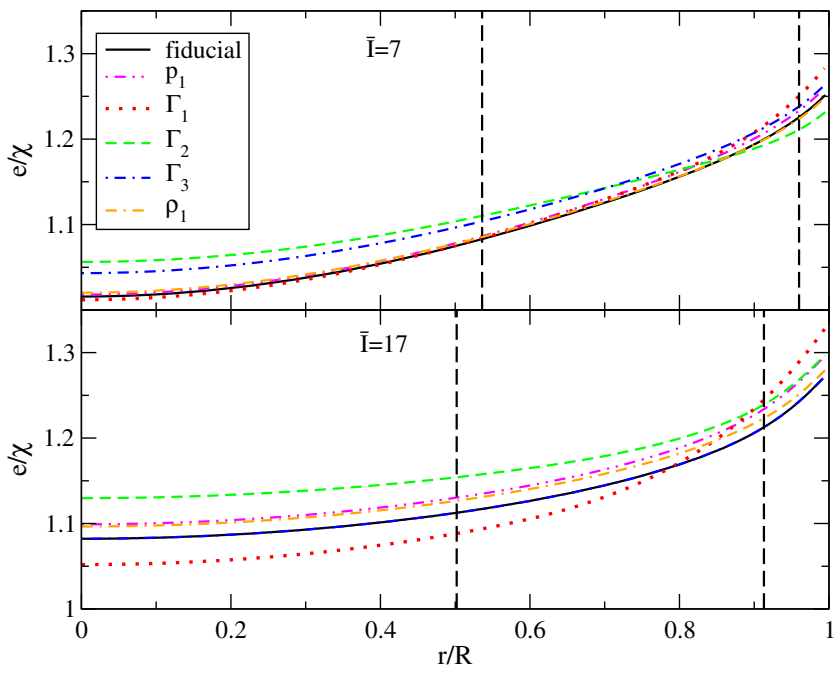

FIG. 2 (color online). Radial profile of the stellar eccentricity (normalized by the dimensionless spin parameter $\chi$ ) for slowly rotating NSs with $\bar{I}=7$ (top) and $\bar{I}=17$ (bottom) for the SLy EOS, and with $30 \%$ variations of $p_{1}, \Gamma_{1}, \Gamma_{2}, \Gamma_{3}$ and $\rho_{1}$. The meaning of vertical lines are the same as in Fig. 1. Notice that since we are working within the slow-rotation approximation, $e / \chi$ does not depend on the NS spin. Observe that the eccentricity varies by $\sim 10 \%$ within the region that matters to the universality. 
$[60,61]$ and extracting the eccentricity profile. We find that the eccentricity variation easily exceeds $100 \%$ inside the star, varying much more than in the relativistic, compactstar case. If the elliptical isodensity approximation is (at least in part) responsible for the universality, then we would expect universality to be lost in such stars. We find that this is indeed the case: the $I-Q$ relation is highly sensitive to variations in the opacity law, which we use as a proxy for EOS variation.

\section{B. A phenomenological picture for universality}

These findings suggest that the EOS universality in NSs and QSs arises due to an (approximate) emergent symmetry. Emergent symmetries are common in quantum field theory and condensed matter physics, for example in the study of chiral spin liquids [62]. The main idea here is that as some set of parameters (usually the energy or temperature of the system) are tuned beyond a given threshold, the description of the system acquires an approximate symmetry that is not present in general.

Consider a phase space with coordinates that represent a quantity that characterizes a star, such as its compactness, temperature, magnetic field strength, etc. Consider now a two-dimensional subspace, with one coordinate being the stellar compactness and the other the EOS polytropic index $n$. NSs live in the large compactness and small $n$ region, while regular, noncompact stars live in the smaller compactness and larger $n$ region, as depicted in Fig. 3. As one flows from the noncompact to the relativistic, compact star region

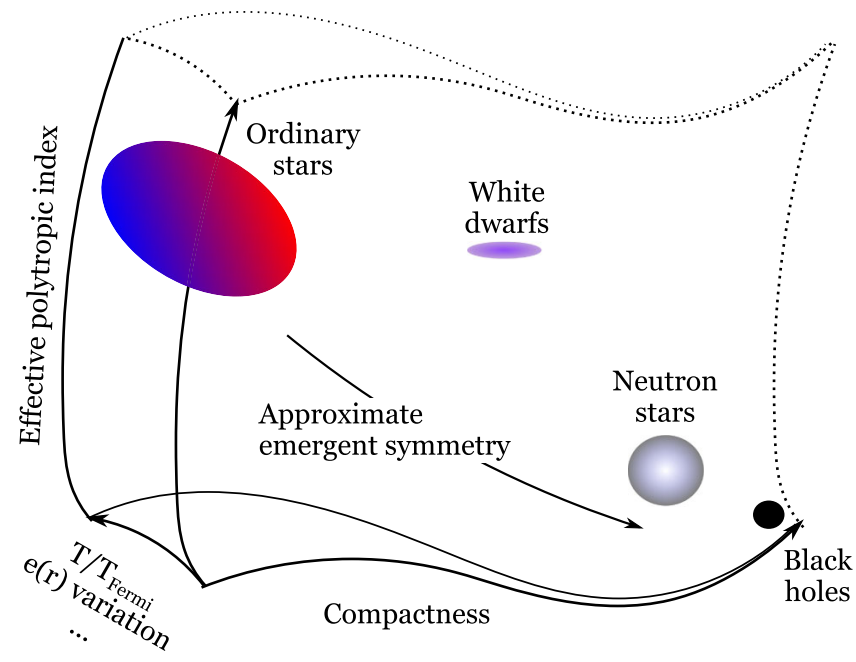

FIG. 3 (color online). Schematic diagram of the stellar phase space. Compact stars live in one corner of this space, while noncompact stars live in another corner. As one flows from the latter to the former along a particular two-dimensional subspace, spanned by compactness $C$ and polytropic index $n$, an approximate symmetry arises: isodensity contours become approximately self-similar. This approximate symmetry is then responsible for the universality in the exterior multipole moments of compact stars. in this projected two-dimensional subspace, eccentricity radial profiles become less variable and nearly constant throughout the star, i.e. a radial remapping leaves the eccentricity profile approximately invariant. This suggests the emergence of an approximate symmetry associated with this isodensity self-similar invariance, which in turn, leads to the universality in the multipole relations for relativistic stars. As one further increases the compactness, one approaches the $\mathrm{BH}$ region in phase space, where the universality becomes exact, as expressed by the no-hair theorems.

\section{Organizations and conventions}

The remainder of this paper describes the main results presented above in much more detail. Section II answers the first question. Focusing mainly on slowly rotating NSs and QSs, we investigate how each piecewise EOS parameter affects the universal I-Q relation. We determine which radial region contributes to $I$ and $Q$ the most and compare the result to the radial profile of the energy density to determine which part of the EOS matters the most. We then compare how similar NS EOSs are within this region. We also construct rapidly rotating NS solutions and see how the rotational and relativistic effects affect the density profile. Section III answers the second question. We relax each of the conditions in the elliptical isodensity approximation and see how they affect the universality for uniformly rotating Newtonian polytropes. Section IV answers the third question. We extract the eccentricity profile of both slowly and rapidly rotating NSs and study the validity of the elliptical isodensity approximation. Section V answers the fourth question. We construct a realistic, differentiallyrotating, noncompact star and investigate the universality on the multipole relations. We also look at the eccentricity profile and compare it with that of realistic relativistic stars. We conclude in Sec. VI by combining these findings into one picture that proposes an emergent approximate symmetry as a possible explanation for the universality. Section VII suggests a few possible directions for future work. All throughout, we use geometric units: $c=1=G$.

\section{THE REGION THAT MATTERS}

What part of the NS interior and what part of the NS EOS contributes the most to the multipole moments of the star, and thus, affects the three-hair relations the most $[25,26,33,36]$ ? In this section, we address this question by studying the multipole moments of NSs characterized be a piecewise EOS. We vary each piece of the EOS and find that the multipole moments are most affected by what is going on in the so-called outer core, i.e. in a shell with inner radius of roughly $0.5 R$ and outer radius $0.95 R$, with $R$ the stellar radius for nonrotating configurations. This region corresponds to densities in roughly the interval $\left(10^{14}-10^{15}\right) \mathrm{g} / \mathrm{cm}^{3}$. In this section, we will provide evidence for these results, mainly focusing on slowly 
rotating NSs and QSs. In the last subsection, we construct rapidly rotating NS solutions and see how the rotational and relativistic effects change the stellar energy density profile.

\section{A. Piecewise polytropic EOSs}

Let us divide the pressure ( $p$ )-rest mass density $(\rho)$ phase space into three regions: the crust, the outer core and the inner core. Figure 4 shows a schematic diagram of this classification. The lowest density region corresponds to the crust, which transitions into the outer core at a density of roughly $10^{14} \mathrm{~g} / \mathrm{cm}^{3}$. The inner and outer cores have higher densities and pressures than the crust, where the transition point is roughly at $10^{14.7} \mathrm{~g} / \mathrm{cm}^{3}$.

Although there is no precise definition of these regions, the choices made above make physical sense. The crustouter core transition is where roughly the lattice of neutronrich nuclei become superfluid neutrons that coexist with type II superconducting protons $[2,63,64]$. The outer and inner core transition, which may or may not exist in some stars depending on their mass, is where the superfluid neutrons condense into pions or transition to a neutron solid or quark matter, or some other phase distinct from a neutron superfluid $[2,63,64]$. The inner core is the region least wellunderstood from nuclear physics experiments, since it is so far-removed from the scales we can probe in the laboratory.

In each of these regions, we model the EOS through polytropic equations. In the crust we will use four polytropes, in the outer and inner core three polytropes. The full EOS is then a piecewise function with 7 polytropes of the form

$$
p(\rho)= \begin{cases}p_{\text {crust }}(\rho), & \left(\rho<\rho_{0}\right), \\ \left(p_{1} / \rho_{1}^{\Gamma_{1}}\right) \rho^{\Gamma_{1}}, & \left(\rho_{0}<\rho<\rho_{1}\right), \\ \left(p_{1} / \rho_{1}^{\Gamma_{2}}\right) \rho^{\Gamma_{2}}, & \left(\rho_{1}<\rho<\rho_{2}\right), \\ \left(\rho_{2} / \rho_{1}\right)^{\Gamma_{2}}\left(p_{1} / \rho_{2}^{\Gamma_{3}}\right) \rho^{\Gamma_{3}}, & \left(\rho_{2}<\rho\right),\end{cases}
$$

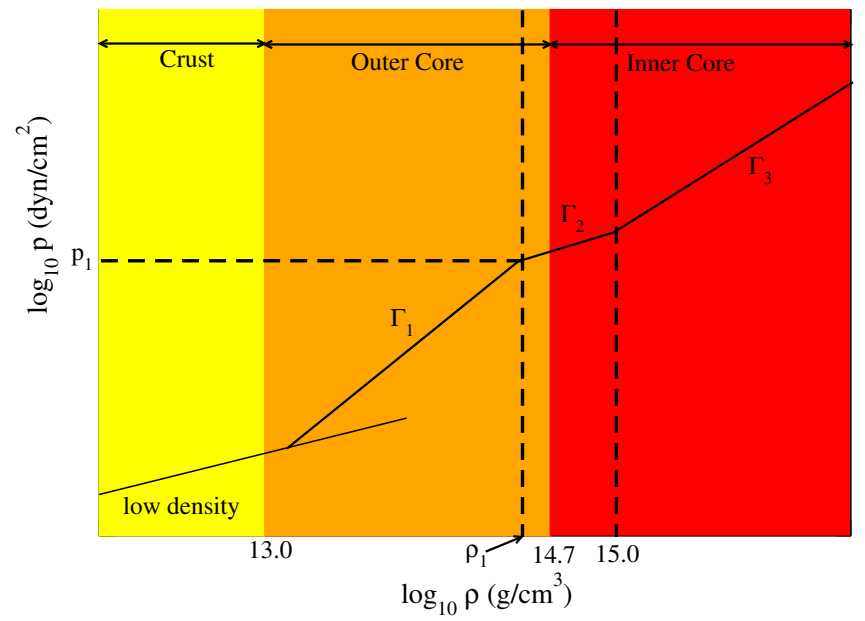

FIG. 4 (color online). Schematic diagram of the piecewise EOS and the different regions of a NS's interior shaded in yellow, orange and red. where $\left(\Gamma_{i}, p_{1}, \rho_{1}\right)$ are free constants, $\rho_{2}=10^{15} \mathrm{~g} / \mathrm{cm}^{3}, \rho_{0}$ is the solution to $p_{\text {crust }}\left(\rho_{0}\right)=p_{1}\left(\rho_{0} / \rho_{1}\right)^{\Gamma_{1}}$, with $p_{\text {crust }}(\rho)$ given in [57]. Such a piecewise EOS depends on 5 free constants $\left(p_{1}, \Gamma_{1}, \Gamma_{2}, \Gamma_{3}, \rho_{1}\right)$, where $\Gamma_{1}$ mainly affects the EOS slope in the outer core, $\Gamma_{2}$ and $\Gamma_{3}$ mainly affect the EOS slope in the inner core, $p_{1}$ is an overall scaling factor that corresponds to the pressure at $\rho=\rho_{1}$ and $\rho_{1}$ changes the transition density of the first two regions. Equation (1) with a fixed $\log _{10} \rho_{1}=14.7$ has been shown to reproduce a great number of realistic EOSs [57], e.g. the SLy EOS [58] is reproduced with $\left(\log _{10} p_{1}, \Gamma_{1}, \Gamma_{2}, \Gamma_{3}\right)=(34.384,3.005$, $2.988,2.851)$ to $1 \%$ accuracy. ${ }^{1}$ The range of values of $\left(p_{1}\right.$, $\left.\Gamma_{1}, \Gamma_{2}, \Gamma_{3}\right)$ that reproduce all known realistic EOSs is, in fact, within approximately $30 \%$ from those of the SLy EOS [57], which is why we choose the latter as our fiducial EOS. We here enlarged the model of [57] by introducing an additional parameter $\left(\rho_{1}\right)$ to increase the degrees of freedom in the variability of the EOS.

For future convenience, let us relate the rest-mass density $\rho$ to the energy density $\epsilon$. From the first law of thermodynamics, the latter is given by [57]

$$
\epsilon_{i}(\rho)=\left(1+a_{i}\right) \rho+\frac{K_{i}}{\Gamma_{i}-1} \rho^{\Gamma_{i}}
$$

in the $i$ th region, where the coefficients $a_{i}$ are given below Eq. (4) of [57]. Notice that it is $\epsilon$ that sources the Einstein equations, while it is $\rho$ that enters the nonrelativistic, Newtonian definition of the multipole moments.

\section{B. Mass-radius and $\bar{I}-\bar{Q}$ relations}

We will study the universal relations between the NS moment of inertia and quadrupole moment in slowly and uniformly rotating, nonmagnetized NSs. We construct these stars by solving the Einstein equations perturbatively in a slow-rotation expansion to quadratic order in spin, following Hartle and Thorne [55,56]. At zeroth order in spin, we calculate the stellar mass $M$ and radius $R$ of the nonrotating configuration. At first order in spin, we extract the moment of inertia

$$
I=\frac{S_{1}}{\Omega}
$$

where $S_{1}$ and $\Omega$ are the spin angular momentum and the angular velocity, respectively. At second order in spin, we calculate the quadrupole moment $Q[55,56]$.

Before looking at $I$ and $Q$, let us investigate how the nonrotating configurations are affected by modifying the piecewise EOS coefficients. Let us then vary these coefficients individually by $30 \%$ from the fiducial SLy values, i.e. we separately set $\log _{10} p_{1}=34.2291$, or $\Gamma_{1}=2.1035$,

\footnotetext{
${ }^{1} \log _{10} \rho_{1}$ and $\log _{10} p_{1}$ mean $\log _{10}\left[\rho_{1} / 1\left(\mathrm{~g} / \mathrm{cm}^{3}\right)\right]$ and $\log _{10}\left[p_{1} / 1\left(\mathrm{dyn} / \mathrm{cm}^{3}\right)\right]$, respectively.
} 


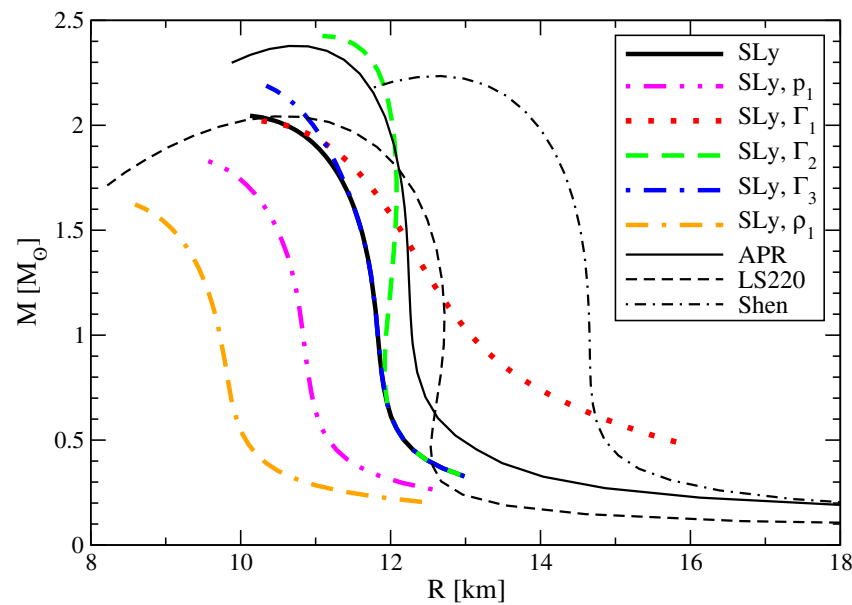

FIG. 5 (color online). Mass-radius relation for the fiducial SLy EOS and that obtained by varying $p_{1}, \Gamma_{1}, \Gamma_{2}, \Gamma_{3}$ and $\rho_{1}$ by $30 \%$. Notice that such a variation has a large impact on the mass-radius relation. We also present the mass-radius relation for the APR, LS220 and Shen EOSs for reference.

or $\Gamma_{2}=3.8844$, or $\Gamma_{3}=3.7063$, or $\log _{10} \rho_{1}=14.8139$, while keeping the other parameters equal to their SLy values. These variations are consistent with the range of variability associated with the different EOSs [57]. Figure 5 shows the mass-radius relation for various modified SLy EOSs, together with the mass-radius relation for the APR [65], LS220 [66] and Shen [67,68] EOSs. We impose a neutrino-less, beta-equilibrium condition for the latter two EOSs. Observe that $\Gamma_{3}$ and $\Gamma_{2}$ only affect the relation for $M>1.7 M_{\odot}$ and $M>0.6 M_{\odot}$, respectively, while $\Gamma_{1}$ has a larger effect on the lower mass region. Since $p_{1}$ and $\rho_{1}$ affect the overall magnitude of the EOS, they scale the mass-radius relation rather than change its shape.

Let us now study how the $\bar{I}-\bar{Q}$ universal relations are affected by changing the piecewise polytropic coefficients. We work with the dimensionless quantities

$$
\bar{I} \equiv \frac{I}{M^{3}}, \quad \bar{Q} \equiv-\frac{Q}{M^{3} \chi^{2}},
$$

where the dimensionless spin parameter is defined to be

$$
\chi \equiv \frac{S_{1}}{M^{2}} .
$$

For reference, a value of $\bar{Q}=1$ and 10 roughly correspond to a nonrotating star mass of $2 M_{\odot}$ and $1 M_{\odot}$, respectively, using the SLy EOS.

The left panel of Fig. 6 shows the fractional difference on the $\bar{I}-\bar{Q}$ relation relative to the fiducial EOS due to changing the piecewise polytrope coefficients. For stars with $M>1.8 M_{\odot}, \Gamma_{2}$ affects the $\bar{I}-\bar{Q}$ relation the most, whereas when $M<1.8 M_{\odot}, \Gamma_{1}$ affects the relation the most, in both cases to $\mathcal{O}(1 \%)$. Such a small fractional difference is precisely why the universality is approximate and it is consistent with that reported in $[25,26]$. Modifications to $p_{1}, \Gamma_{3}$ and $\rho_{1}$ affect the $\bar{I}-\bar{Q}$ relations the least, by only $0.1 \%$. In particular, $p_{1}$ and $\rho_{1}$ essentially scale the EOS, which does not affect $\bar{I}$ and $\bar{Q}$ as a function of $C$ [26]; the $\bar{I}-\bar{Q}$ relation is insensitive to variations of these parameters.

The right panel of Fig. 6 presents the fractional difference of the $\bar{I}-\bar{Q}$ relation at $\bar{Q}=2.5$ (top) and $\bar{Q}=8$ (bottom) from the fiducial SLy EOS against various
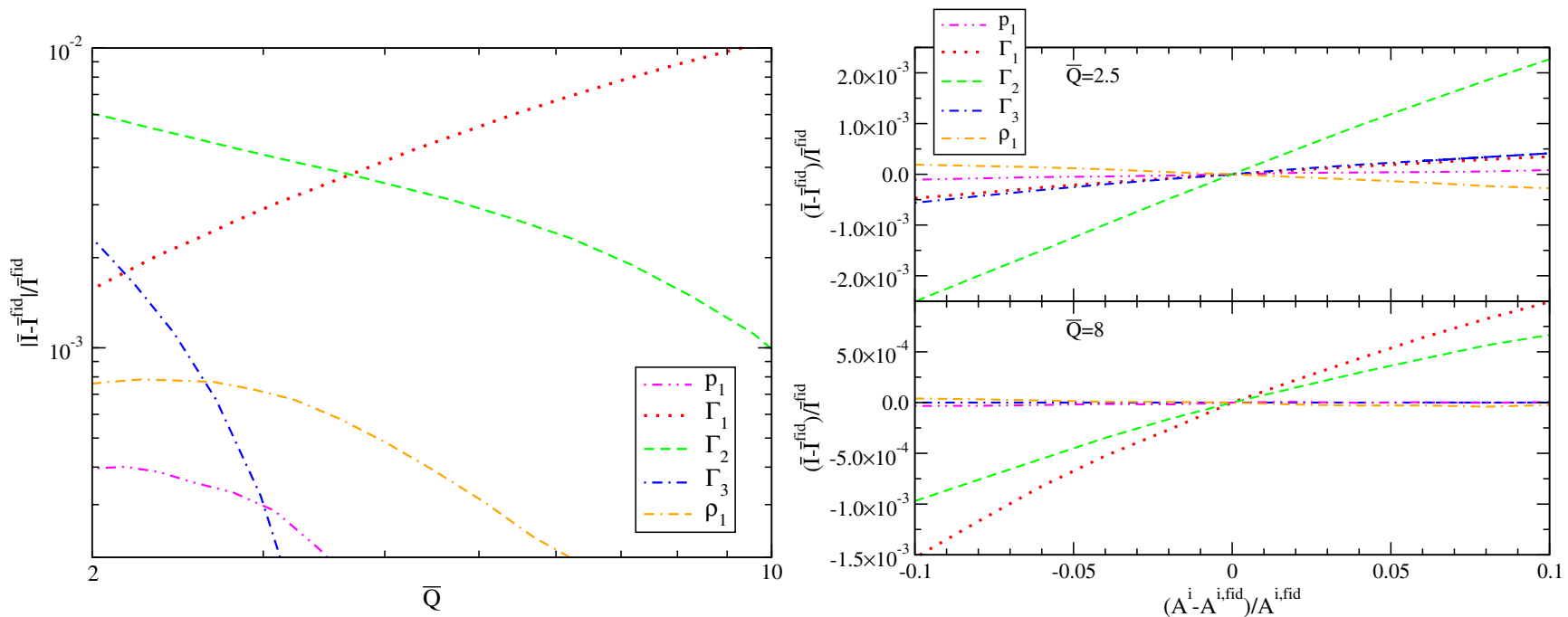

FIG. 6 (color online). Left: Fractional difference on the $\bar{I}-\bar{Q}$ relation between the varied and fiducial SLy EOSs. For the former, we vary $p_{1}, \Gamma_{1}, \Gamma_{2}, \Gamma_{3}$ and $\rho_{1}$ by $30 \%$. Right: (Top) Fractional difference of the $\bar{I}-\bar{Q}$ relation at $\bar{Q}=2.5$ with varied piecewise EOS parameters from those of the fiducial SLy EOS. The horizontal axis represents the fractional deviation of $A^{i}=\left(p_{1}, \Gamma_{1}, \Gamma_{2}, \Gamma_{3}, \rho_{1}\right)$. Observe that the fractional difference scales almost linearly with the piecewise EOS parameters. (Bottom) Same as the top panel, but for $\bar{Q}=8$. 
piecewise EOS parameters, $A^{i}=\left(p_{1}, \Gamma_{1}, \Gamma_{2}, \Gamma_{3}, \rho_{1}\right)$. Observe that the fractional difference scales almost linearly with the EOS parameters. This figure shows that changing the EOS parameters by $10 \%$ only modifies the relation by $\sim 0.2 \%$ at most. We have checked that the fractional difference in the relation when varying $\Gamma_{1}$ and $\Gamma_{2}$ simultaneously (the two most important parameters regarding universality) is essentially the same as varying $\Gamma_{1}$ and $\Gamma_{2}$ separately and then adding their fractional difference linearly.

The right panel of Fig. 6 also shows that decreasing the EOS slopes $\Gamma_{i}$ from the fiducial values affects the universality more than if we increase the slopes. Increasing $\Gamma_{i}$ corresponds to decreasing the polytropic index $n$, since $\Gamma=1+1 / n$. For example, the SLy values of $\left(\Gamma_{1}, \Gamma_{2}\right.$, $\left.\Gamma_{3}\right)=(3.005,2.988,2.851)$ correspond to $\left(n_{1}, n_{2}, n_{3}\right)=$ $(0.499,0.503,0.540)$, and thus, decreasing $\Gamma_{i}$ corresponds to increasing $n$ above 0.5 , and vice-versa. Strange quark stars are well-modeled by polytropes with index $n \sim 0$, while white dwarfs are well modeled with polytropes of index $n>2$. Thus, as we increase $\Gamma_{i}$ we make the star look more like a QS, while when we decrease $\Gamma_{i}$, it looks more like a white dwarf. Notice, interestingly, that the universality is affected more as we approach the white-dwarf branch than as we approach the QS branch. We think this is because as we approach this branch, the elliptical isodensity approximation becomes less valid, which significantly affects the universality, as we will see later in this paper.

\section{Radial integrand of the NS and QS moment of inertia and quadrupole moment}

In order to understand which part of the stellar interior region affects the universal relations the most, let us look at the radial profile of the integrand of the moment of inertia for a fixed $\bar{I}$. For slowly rotating stars in full GR, $I$ is given in integral form as [55]

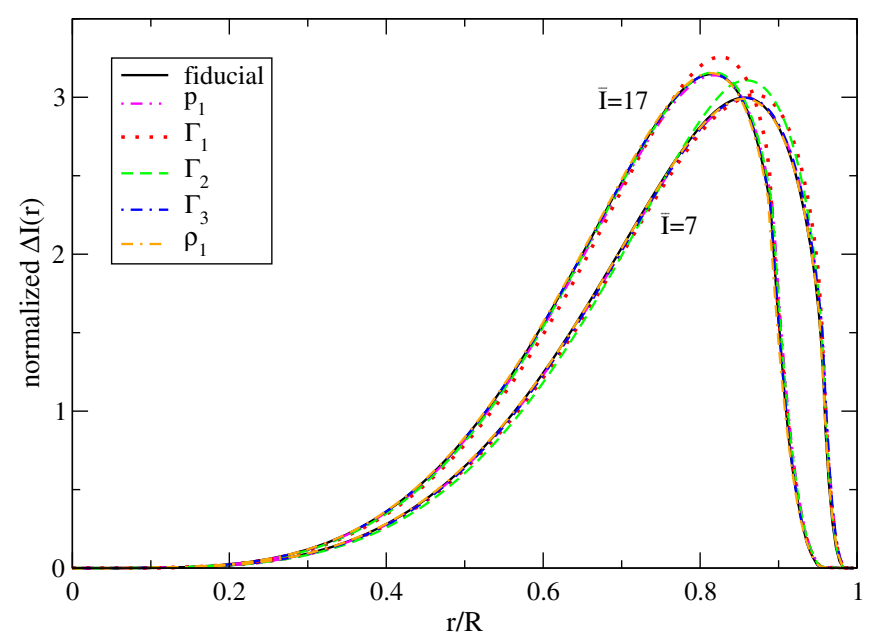

$$
I=\int_{0}^{R} \Delta I(r) d r, \quad \Delta I(r) \equiv-\frac{2}{3} r^{3} \frac{d j}{d r} \frac{\omega(r)}{\Omega},
$$

where $R$ is the stellar radius for a nonrotating configuration,

$$
j(r) \equiv e^{-[\nu(r)+\Lambda(r)] / 2},
$$

and $\nu, \Lambda$ and $\omega$ are metric functions related to the linearin-spin line element via

$$
\begin{aligned}
d s^{2}= & -e^{\nu(r)} d t^{2}+e^{\Lambda(r)} d r^{2} \\
& +r^{2}\left\{d \theta^{2}+\sin ^{2} \theta[d \phi-[\Omega-\omega(r)] d t]^{2}\right\} .
\end{aligned}
$$

Equation (6) reduces to [55]

$$
I^{N}=\frac{8 \pi}{3} \int_{0}^{R} r^{4} \rho(r) d r
$$

in the Newtonian limit.

Figure 7 shows $\Delta I$ as a function of fractional radius for NSs (left) with the fiducial SLy EOS and EOS parameters that deviate by $10 \%$ from the fiducial EOS, and for QSs (right) with three different QS EOSs, with $\bar{I}=7$ and $\bar{I}=17$ held fixed. We normalized the integrands such that the area in Fig. 7 is unity. Observe that the integrand is dominated by the region within $50 \%-95 \%$ of the total radius. Observe also that the integrands are almost unaffected by the variation of the EOS parameters for a fixed $\bar{I}$. These results extend and confirm those found in [26], where a related integrand was investigated in the Newtonian limit for a fixed NS compactness.

In order to quantify which part of the stellar interior contributes the most to the integrand, we identify the region, delimited by $r_{\min }$ and $r_{\max }$, inside which the integrand integrates to $90 \%$ of the total $I$ :

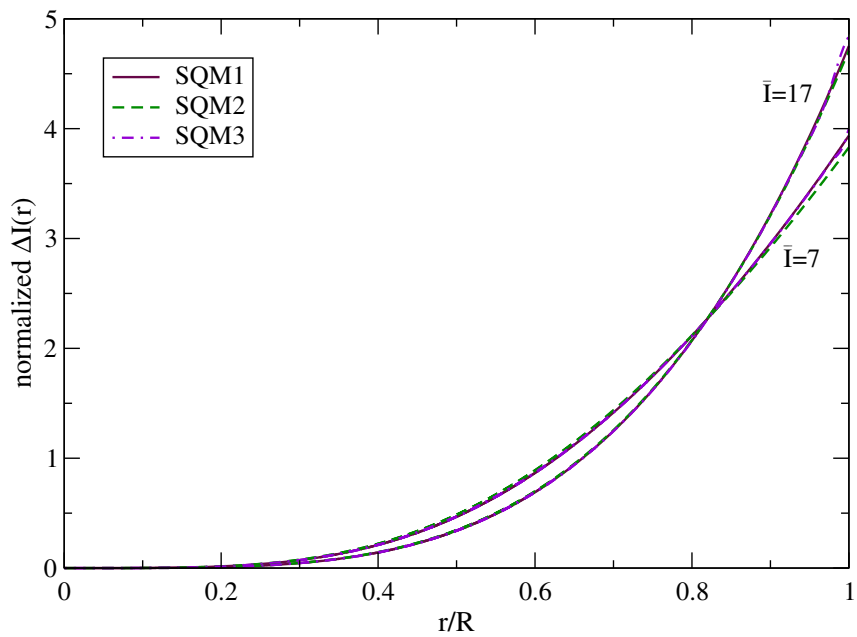

FIG. 7 (color online). The radial profile of the normalized integrand of $I$ at $\bar{I}=7$ and $\bar{I}=17$ for NSs with the fiducial SLy EOS and QSs with three different QS EOSs [69] (right). The other curves in the left panel correspond to varying the EOS parameters by $10 \%$ from the fiducial ones. Observe that the integrands are not very sensitive to the variation of the EOS. 


$$
\int_{r_{\min }}^{r_{\max }} \Delta I(r) d r=0.9 \int_{0}^{R} \Delta I(r) d r
$$

with the symmetry constraint that $\Delta I\left(r_{\min }\right)=\Delta I\left(r_{\max }\right)$. In the NS case with the fiducial SLy EOS, we find $\left(r_{\min }, r_{\max }\right)=(0.54,0.96) R$ and $(0.50,0.91) R$ for $\bar{I}=7$ and $\bar{I}=17$, respectively. In the QS case, $\left(r_{\min }, r_{\max }\right)=$ $(0.58,1) R$ and $\left(r_{\min }, r_{\max }\right)=(0.62,1) R$ for $\bar{I}=7$ and $\bar{I}=17$, respectively. In the latter case, the integrand increases monotonically as a function of $r / R$ because the density does not drop to zero at the stellar surface, as shown in Fig. 8. QSs correspond to nearly constant density stars, especially when $\bar{I}$ is large, where the stellar compactness is relatively small. These results confirm that $\bar{I}$ is mostly affected by the region in the approximate range $50 \%-95 \%$ and $60 \%-100 \%$ of the stellar radius in the NS and QS cases, respectively.

One can also consider the radial profile of the integrand of $Q$. When the stellar eccentricity is constant throughout the star, $Q$ is given by $Q \propto e^{2} \int \rho r^{4} d r$ (see Eqs. (5) and (7) in [36]) for Newtonian, slowly rotating polytropes. In reality, eccentricity is a function of radius and one has to include $e(r)^{2}$ in the radial integral. Therefore, the integrand of $Q$ is $\Delta Q \sim \rho(r) r^{4} e(r)^{2}$, modulo an overall constant. We checked that the radial profile of such integrand is almost identical to the one of $\Delta I(r)$ in Fig. 7. This means that both $\bar{I}$ and $\bar{Q}$ are mostly affected by the region in the range $50 \%-95 \%$ of the radius.

That the $\bar{I}-\bar{Q}$ relations are dominated by the EOS in the region roughly between $50 \%$ and $95 \%$ of the NS's total radius was first pointed out in $[25,26]$. Unfortunately, that paper referred to this region as the "outer-layers" of the NSs, which has recently been taken to mean the NS crust.

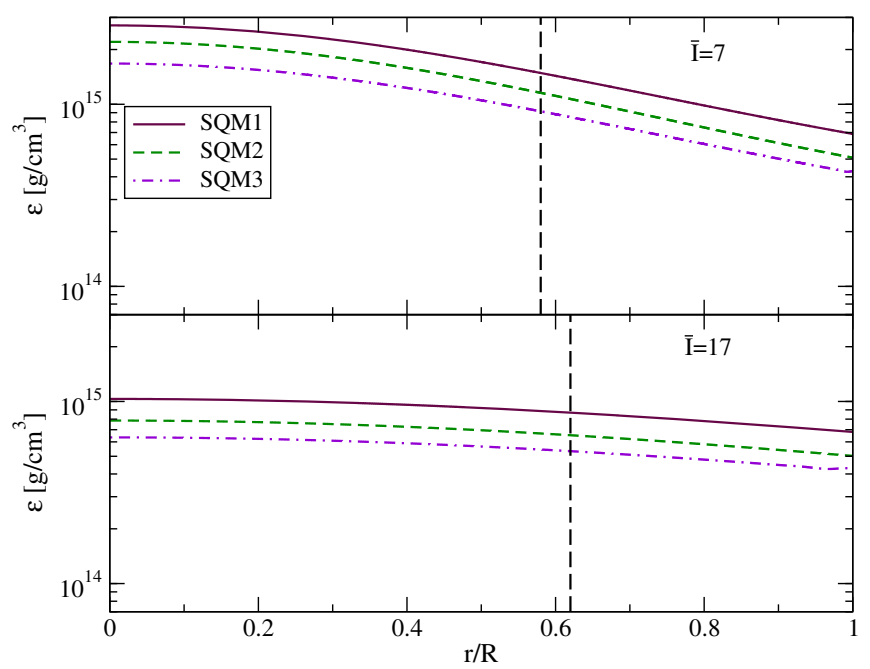

FIG. 8 (color online). Energy density profile (same as Fig. 1) but for QSs. Observe that the density is almost constant throughout the star and nonvanishing at the stellar surface. The region above the vertical dashed line gives a $90 \%$ contribution to the QS moment of inertia and quadrupole moment.

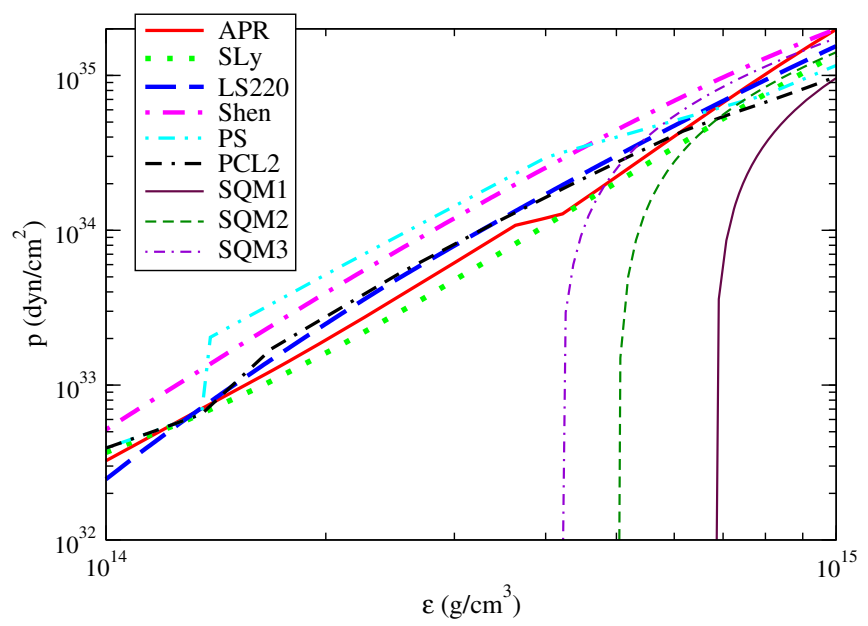

FIG. 9 (color online). EOS in the $(p, \epsilon)$ region that dominates the calculation of the moment of inertia.

Clearly the region $r / R \in(0.50,0.95)$ does not comprise the NS crust, but rather the outer core and the very outer part of the inner core.

With this at hand, we can now determine the $(p, \epsilon)$ region of the EOS that dominates the universal relations. Figures 1 and 8 show the energy density profile of NSs and QSs, respectively, with $\bar{I}=7$ (top) and $\bar{I}=17$ (bottom) for various modified EOSs, together with $r_{\min } / R$ and $r_{\max } / R$ plotted as vertical dashed lines. Recall that larger values of $\bar{I}$ correspond to smaller stellar compactnesses $C=M / R$. The figure shows that $\bar{I}$ and $\bar{Q}$ are most affected by the EOS in the range $\epsilon=\left(10^{14}-10^{15}\right) \mathrm{g} / \mathrm{cm}^{3}$ and $\epsilon=(4 \times$ $\left.10^{14}-1.5 \times 10^{15}\right) \mathrm{g} / \mathrm{cm}^{3}$ in the NS and QS cases, respectively.

One possible explanation for the approximate EOS universality is that all EOSs are approximately the same in this density region. We found in Sec. II B that the EOS slope is more important than the overall EOS magnitude. Therefore, one needs to look at the variation in the EOS slope in the density region that matters, as shown in Fig. 9. In order to quantify this variation, we fitted a polytrope of the form $p=\bar{K} \epsilon^{\bar{\Gamma}}$ to several tabulated NS EOSs in this region (see Table I for the best-fit values of $\bar{\Gamma}$ and the estimated

TABLE I. Best-fit value of the EOS slope $\bar{\Gamma}$ for various realistic NS EOSs in the energy density range $10^{14}-10^{15} \mathrm{~g} / \mathrm{cm}^{3}$, where the moment of inertia and quadrupole moment are affected the most.

\begin{tabular}{ll}
\hline \hline \multicolumn{1}{c}{ EOS } & \multicolumn{1}{c}{$\langle\bar{\Gamma}\rangle$} \\
\hline APR [65] & $2.79 \pm 0.016$ \\
SLy [58] & $2.70 \pm 0.037$ \\
LS220 [66] & $2.77 \pm 0.0086$ \\
Shen [67,68] & $2.62 \pm 0.0064$ \\
PS [70] & $2.35 \pm 0.076$ \\
PCL2 [69] & $2.57 \pm 0.064$ \\
\hline \hline
\end{tabular}


errors). Observe that the variation in the slope can be as high as $\sim 17 \%$, but it would increase significantly if one included QSs. Thus, the approximate similarity of the EOS (or its slope) in the density region that matters cannot be the direct explanation for the approximate EOS universality we observe. As we will see in Sec. IV, such $\mathcal{O}(10 \%)$ variation in the EOS slope produces a very small variation in the stellar eccentricity profile, which we propose as the main origin of the approximate universality.

\section{Relativistic and rotational effects on the density profiles}

Do the conclusions derived in the previous subsection continue to hold for relativistic stars with a rapid rotation? In this subsection, we tackle this question, but to keep it simple, we parameterize the EOS through the following polytrope:

$$
p=\bar{K} \epsilon^{1+1 / \bar{n}},
$$

where we recall that $\epsilon$ is the stellar energy density. $\bar{K}$ and $\bar{n}$ are the overall constant and polytropic index, respectively. Notice that $\bar{n}$ is different from $n$ introduced at the end of Sec. II B as the former is the power index for $\epsilon$ while the latter is the one for $\rho$. With this EOS, we now calculate the rotational corrections to the energy density radial profile to see if such effects limit the validity of the arguments posed in the previous subsection. We also study the relativistic effects to see the validity of Newtonian calculations that we will present in the next section.

Let us first concentrate on relativistic modifications to nonrotating configurations. In GR, the equation of hydrostatic equilibrium in spherical symmetry is modified from its Newtonian form to the Tolman-Oppenheimer-Volkov (TOV) equation:

$$
\frac{d p}{d r}=-\frac{m \epsilon}{r^{2}}\left(1+\frac{p}{\epsilon}\right)\left(1+\frac{4 \pi r^{3} p}{m}\right)\left(1-\frac{2 m}{r}\right)^{-1} .
$$

Relativistic corrections arise because all energy densities gravitate (the first two terms) and the geometry is modified (the last term). $m(r)$ in the above equation is related to $\Lambda(r)$ in Eq. (8) via

$$
e^{-\Lambda(r)}=1-\frac{2 m(r)}{r},
$$

where we use Schwarzschild-like coordinates.

The TOV equation can be written in a dimensionless form if one makes the following substitutions: $\epsilon=\epsilon_{c} \bar{\vartheta}^{\bar{n}}$, $r=\alpha \xi, p=\bar{K} \epsilon_{c}^{1+1 / \bar{n}} \bar{\vartheta}^{\bar{n}+1}$ and $m=\epsilon_{c} \alpha^{3} \bar{m}$, where $p_{c}$ and $\epsilon_{c}$ are the pressure and energy density at the stellar center and $\alpha^{2} \equiv(\bar{n}+1) \bar{K} \epsilon_{c}^{1 / \bar{n}-1}$. The new dimensionless equation has the form, ${ }^{2}$

\footnotetext{
${ }^{2}$ An equivalent equation is found in [71].
}

$$
\begin{aligned}
\frac{d \bar{\vartheta}}{d \xi}= & -\frac{\bar{m}}{\xi^{2}}(1+\lambda \bar{\vartheta})\left(1+\lambda \frac{4 \pi \bar{\vartheta}^{\bar{n}+1} \xi^{3}}{\bar{m}}\right) \\
& \times\left(1-2(\bar{n}+1) \lambda \frac{\bar{m}}{\xi}\right)^{-1},
\end{aligned}
$$

where $\lambda \equiv p_{c} / \epsilon_{c}$ is a measure of how relativistic a particular configuration is, and thus, it parametrizes the deviations from the Newtonian approximation. One recovers the (Newtonian) Lane-Emden (LE) equation when $\lambda=0$, with only a difference in scales by a factor of $\sqrt{4 \pi}$ due to different definitions of $\alpha$.

Figure 10 shows the energy density profiles for Newtonian models $(\lambda=0)$ and relativistic models with $\lambda=$ $[0,0.5]$ in increments of 0.1 , for EOSs with polytropic index $\bar{n}=0.5,1$, and 1.5 . The $\lambda=0.5$ case corresponds approximately to the maximum mass model for these polytropes. Observe that as relativistic effects become stronger, the density profiles become more centrally condensed. This effect is more pronounced for higher $\bar{n}$ values, which is consistent with [71].

Let us now study rotational modifications to the energy density profile. To do so, we construct sequences of rotating models using the RNS code [59]. Calculations are performed in quasi-isotropic coordinates, but we will present the results in Schwarzschild-like coordinates so that one can compare them to the slow-rotation results presented earlier. Specifically, for different central densities, starting from values which correspond to Newtonian stars

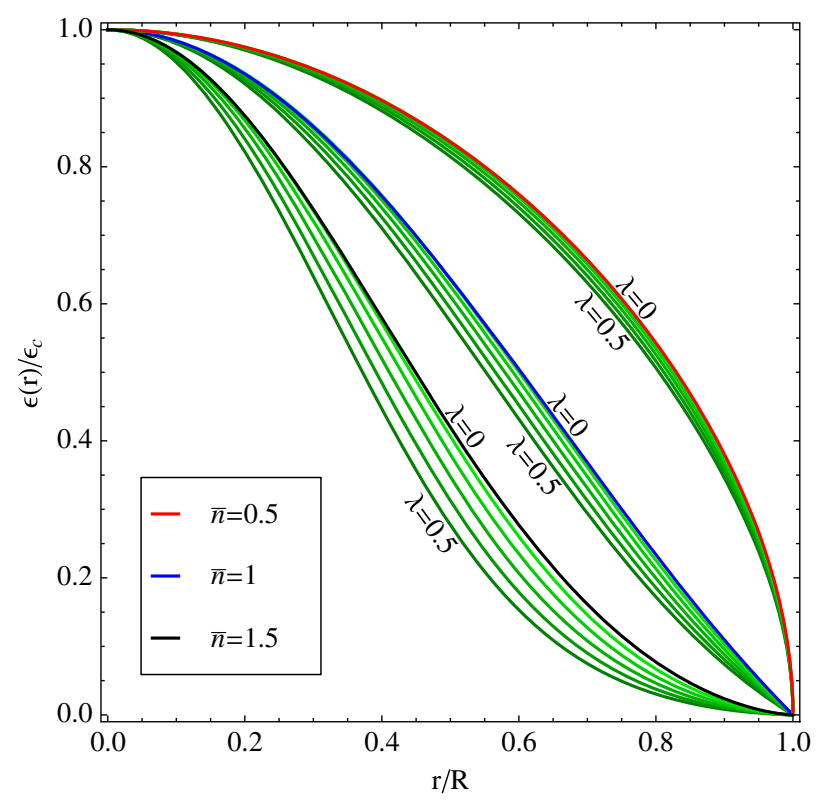

FIG. 10 (color online). Energy density profile for nonrotating polytropes in the Newtonian limit $(\lambda=0)$ and with relativistic corrections using $\lambda=[0,0.5]$ in increments of 0.1 from top to bottom and various polytropic indices $\bar{n}$. Observe how relativistic effects make the profiles more centrally-condensed. 
up to values that correspond to the relativistic models of maximum mass, we construct sequences of rotating polytropes with rotation rates up to the Kepler (massshedding) limit.

Figure 11 shows the energy density profile for $\bar{n}=0.5$ (top panels) and 1 (bottom panels) with various rotation rates. Rotation can change density profiles of nonrotating configurations at most by $\sim 30 \%$. Observe that rotation creates more centrally-condensed configurations. This effect is not very strong for Newtonian models but becomes more prominent for relativistic ones. One might think that the centrifugal force arising from rotation would make objects less centrally condensed, rather than more centrally condensed. However, the profiles we are studying here are normalized density as a function of normalized equatorial radius, $r_{\mathrm{Eq}} / R_{\mathrm{Eq}}$. Along the equatorial plane, all points are pushed outward by rotation. The amount by which they are pushed out increases with radius, in agreement with the eccentricity increasing with radius. Thus, the denominator $R_{\mathrm{Eq}}$ increases faster than the numerator $r_{\mathrm{Eq}}$, so in terms of the ratio $r_{\mathrm{Eq}} / R_{\mathrm{Eq}}$, a value of given normalized density
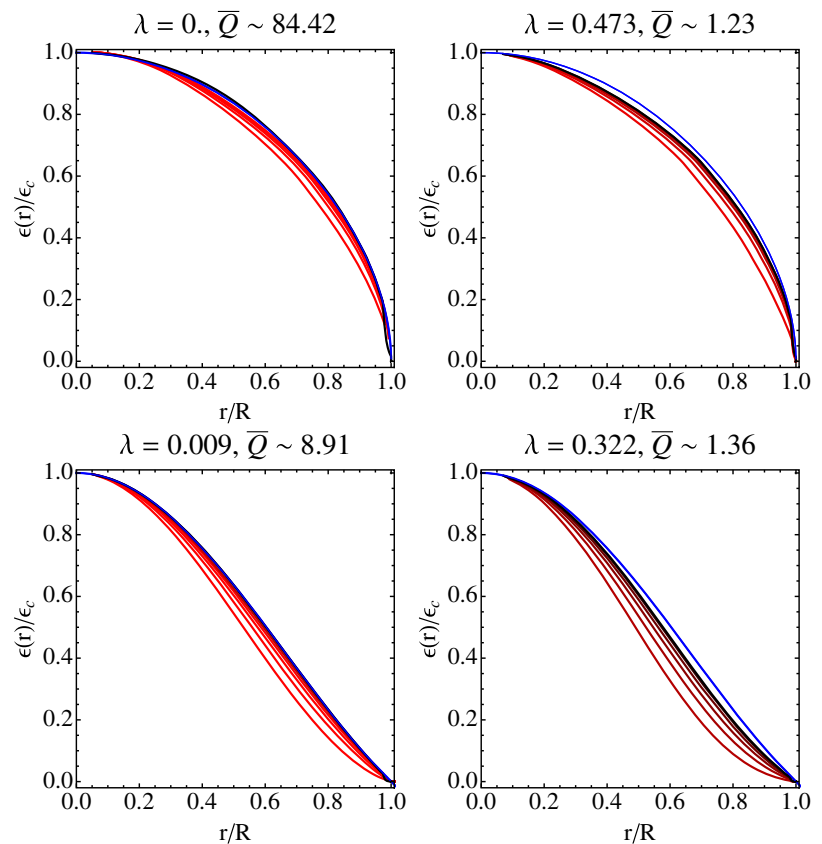

FIG. 11 (color online). The energy density profile for polytropes with an index $\bar{n}=0.5$ (top panels) and 1 (bottom panels), for two extreme values of $\lambda$ and various rotation rates. The blue and black curves are the Newtonian Lane-Emden and relativistic TOV models of nonrotating configurations, respectively. Five red curves present the relativistic rotating models with rotation of $\chi=4.19,7.52,10.05,12.25,14.21$ (Newtonian stars have large radius, and hence have large (dimensionless) angular momentum that can easily exceed unity.) for $\lambda=0$ (top, left), $\chi=0.25,0.45$, $0.58,0.69,0.76$ for $\lambda=0.473$ (top, right), $\chi=0.49,0.87,1.15$, $1.38,1.56$ for $\lambda=0.009$ (bottom, left) and $\chi=0.2,0.35,0.46$, $0.54,0.59$ for $\lambda=0.322$ (bottom, right) from top to bottom. moves inwards, i.e. the profile becomes more centrally condensed.

We have then seen that relativistic corrections and rotational corrections do modify the density profiles of stellar configurations. These modifications, however, are of $\mathcal{O}(10 \%)$ relative to the results obtained in the Newtonian, nonrelativistic limit. Thus, these corrections do not alter the reasoning presented in the previous subsection. For example, in Fig. 1 and 8 we presented the density profile of slowly rotating NSs and QSs. For rapidly rotating models, these profiles would change by a relative factor of $\mathcal{O}(10 \%)$, but the boundaries of the radial and density regions that matter the most for the existence of approximate universality would essentially not be modified.

\section{RELAXING THE ELLIPTICAL ISODENSITY APPROXIMATION}

In the previous section, we established that the region that matters the most regarding universality is the outer core of the star. With this knowledge at hand, let us try to understand what approximate symmetries are present that could be responsible for the universality observed. To do so, we will work in the nonrelativistic, Newtonian limit, as then we can do all calculations analytically and understand the symmetries more clearly. Relativistic corrections were already studied in Sec. II D and in [35].

One of the key approximations used to derive the nohair-like relations for NSs and QSs in the nonrelativistic limit [36] was the elliptical isodensity approximation [54]. This approximation has three main ingredients:

(1) Self-similar isodensity condition. That the density profile is a sequence of self-similar surfaces with the same, constant stellar eccentricity

$$
e=e_{0}=\text { const. }
$$

This implies that the radius- and polar angledependent density profile can be approximated as a function of a single radial variable, $\tilde{r}$, i.e. $\rho(r, \theta)=\rho(\tilde{r})$. Although we have introduced an eccentricity here, we have not yet restricted the isodensity contours to ellipsoids.

(2) Elliptical condition. That the shape of the isodensity surfaces is an ellipsoid. This implies that there exists a change of coordinates, from spherical to elliptical, such that the moment-integrals separate:

$$
R_{*}^{\text {ellip }}(\theta)=\left(\frac{\sin ^{2} \theta}{a_{1}^{2}}+\frac{\cos ^{2} \theta}{a_{3}^{2}}\right)^{-1 / 2},
$$

where $R_{*}^{\text {ellip }}$ denotes the elliptical stellar surface and $a_{1}$ and $a_{3}$ are the semi-major and semi-minor axis of the ellipsoid. This condition, in particular, excludes triaxial surfaces. 
(3) Spherical Density Profile Condition. That once we have transformed to the radial coordinate $\tilde{r}$, the rescaled density $\vartheta=\left(\rho / \rho_{c}\right)^{1 / n}$, where $\rho_{c}$ is the central density, is a LE function, i.e. the rescaled density for the nonrotating Newtonian polytropes:

$$
\vartheta(\tilde{r})=\vartheta_{\mathrm{LE}}(\tilde{r}) .
$$

In order to understand which condition is responsible for the approximate EOS universality observed in [36], we systematically relax each of them and assess their relative importance. We find that the particular shape of the selfsimilar surfaces [condition (2)] does not impact the universality at all. On the other hand, the self-similar assumption [condition (1)] and the spherical density approximation [condition (3)] do affect the universality dramatically. We will see below that breaking either of these conditions can destroy the approximate EOS universality.

\section{A. Universal relations with the elliptical isodensity approximation}

Before we begin to break the conditions of the elliptical isodensity approximation, let us review how the approximate EOS universal no-hair relations are derived in the Newtonian limit. Consider a uniformly rotating, unmagnetized and cold Newtonian star with a polytropic EOS, given by

$$
p=K \rho^{1+1 / n} .
$$

The mass and current multipole moments in the nonrelativistic, Newtonian limit are [72]:

$$
\begin{aligned}
& M_{\ell}=2 \pi \int_{-1}^{1} d \mu \int_{0}^{R_{*}(\mu)} d r \rho(r, \mu) P_{\ell}(\mu) r^{\ell+2}, \\
& S_{\ell}=\frac{4 \pi \Omega}{\ell+1} \int_{-1}^{1} d \mu \int_{0}^{R_{*}(\mu)} d r \rho(r, \mu) \frac{d P_{\ell}(\mu)}{d \mu}\left(1-\mu^{2}\right) r^{\ell+3},
\end{aligned}
$$

where $\mu=\cos \theta, R_{*}(\mu)$ is the stellar surface, $P_{\ell}(\mu)$ are Legendre polynomials and $\Omega$ is the star's angular frequency. For future convenience, let us introduce here the dimensionless multipole moments

$$
\begin{aligned}
\bar{M}_{2 \ell+2} & =(-1)^{\ell+1} \frac{M_{2 \ell+2}}{M^{2 \ell+3} \chi^{2 \ell+2}}, \\
\bar{S}_{2 \ell+1} & =(-1)^{\ell} \frac{S_{2 \ell+1}}{M^{2 \ell+2} \chi^{2 \ell+1}},
\end{aligned}
$$

where $\chi \equiv S_{1} / M^{2}$. Notice that $M_{2}=Q$ is the (mass) quadrupole moment, while $S_{1}$ is the (current) dipole moment and $M_{0}=M$ is the (mass) monopole.
Let us begin by re-deriving the first universal relation of [36], using only the self-similar isodensity condition [condition (1)]. This assumption allows us to separate the moment integrals into radial and angular parts. Introducing the coordinate system $x^{i}=\tilde{r} \Theta(\mu) n^{i}$, with $n^{i}=(\sin \theta \cos \phi, \sin \theta \sin \phi, \cos \theta)$, for some function $\Theta$ of $\mu$ only (due to the self-similar isodensity condition), we find

$$
M_{\ell}=2 \pi I_{\ell, 3} R_{\ell}, \quad S_{\ell}=\frac{4 \pi \ell}{2 \ell+1} \Omega \delta I_{\ell} R_{\ell+1},
$$

where the radial and angular integrals are given by

$$
R_{\ell}=\int_{0}^{a_{1}} \rho(\tilde{r}) \tilde{r}^{\ell+2} d \tilde{r}, \quad I_{\ell, k}=\int_{-1}^{1} \Theta(\mu)^{\ell+k} P_{\ell}(\mu) d \mu,
$$

and $\delta I_{\ell}=I_{\ell-1,5}-I_{\ell+1,3}$, with $a_{1}$ the stellar semi-major axis, which satisfies $a_{1} \Theta(\mu)=R_{*}(\mu)$. One can then immediately derive the first universal relation by taking the ratio of the moments:

$$
\frac{\bar{M}_{2 \ell+2}}{\bar{S}_{2 \ell+1}}=\left[\begin{array}{ll}
\frac{4 \ell+3}{6 \ell+3} \frac{\delta I_{1}}{\delta I_{2 \ell+1}} & \frac{I_{2 \ell+2,3}}{I_{2,3}}
\end{array}\right] \bar{M}_{2} .
$$

This relation does not depend on the polytropic index $n$ since it does not explicitly depend on $R_{\ell}$, but it does depend on the spin (or equivalently on $e$ ) in general.

Let us now use the elliptical condition [condition (2)] in Eq. (25). Doing so, the $\Theta(\mu)$ function is uniquely given by [36]

$$
\Theta(\mu) \equiv \sqrt{\frac{1-e^{2}}{1-e^{2}\left(1-\mu^{2}\right)}},
$$

where $e$ is the eccentricity of the ellipsoids. Using this, the first universal relation becomes

$$
\frac{\bar{M}_{2 \ell+2}}{\bar{S}_{2 \ell+1}}=\bar{M}_{2} .
$$

This is the final form of the first universal relation, which has now become not only EOS-independent but also spinindependent. Notice that the third condition of the elliptical isodensity approximation was never needed.

Let us now consider the second universal relation. To derive this, let us first use the self-similar isodensity condition [condition (1)] and define

$$
R_{\ell}=\rho_{c}\left(\frac{a_{1}}{\xi_{1}}\right)^{\ell+3} \mathcal{R}_{n, \ell},
$$

where $\xi=\left(\xi_{1} / a_{1}\right) \tilde{r}$ is a dimensionless radius with $\xi=\xi_{1}$ the surface of the star and 


$$
\mathcal{R}_{n, \ell}=\int_{0}^{\xi_{1}}[\vartheta(\xi)]^{n} \xi^{\ell+2} d \xi,
$$

with $\vartheta(\xi)=\left(\rho / \rho_{c}\right)^{1 / n}$. We can reexpress $\rho_{c}$ in terms of the mass monopole [Eq. (23)] to find

$$
\rho_{c}=\frac{M}{2 \pi I_{0,3}}\left(\frac{\xi_{1}}{a_{1}}\right)^{3} \frac{1}{\mathcal{R}_{n, 0}} .
$$

Similarly, we can reexpress $a_{1} / \xi_{1}$ in terms of $S_{2 \ell+1} / S_{1}$. After doing so, we find the second universal relation,

$$
\bar{M}_{2 \ell+2}=\bar{A}_{n, \ell}\left(\bar{S}_{2 \ell+1}\right)^{1+1 / \ell},
$$

where we have defined the coefficient

$$
\bar{A}_{n, \ell}=\left(\frac{4 \ell+3}{6 \ell+3}\right)^{1+1 / \ell} \frac{I_{2 \ell+2,3}}{I_{0,3}}\left(\frac{\delta I_{1}}{\delta I_{2 \ell+1}}\right)^{1+1 / \ell} \tilde{\mathcal{R}}_{n, \ell},
$$

with

$$
\tilde{\mathcal{R}}_{n, \ell} \equiv \frac{\mathcal{R}_{n, 2}^{1+1 / \ell}}{\mathcal{R}_{n, 0} \mathcal{R}_{n, 2 \ell+2}^{1 / \ell}} .
$$

Observe that $\bar{A}_{n, \ell}$ depends on the angular integral, and hence it depends on the spin in general. Notice that we have only applied the first condition of the elliptical isodensity approximation to derive Eqs. (31)-(33).

Let us now use the second and third conditions. If one uses the second one through Eq. (26), then Eq. (32) reduces to

$$
\bar{A}_{n, \ell}=\frac{(2 \ell+3)^{1 / \ell}}{3^{(1+1 / \ell)}} \tilde{\mathcal{R}}_{n, \ell} .
$$

On top of this, if one assumes the third condition through Eq. (17), Eq. (33) reduces to

$$
\tilde{\mathcal{R}}_{n, \ell}^{\mathrm{LE}} \equiv \frac{\mathcal{R}_{n, 2}^{\mathrm{LE} 1+1 / \ell}}{\left|\vartheta_{\mathrm{LE}}^{\prime}\left(\xi_{1}\right)\right| \xi_{1}^{2} \mathcal{R}_{n, 2 \ell+2}^{\mathrm{LE}} 1 / \ell},
$$

where we used the LE equation,

$$
\frac{1}{\xi^{2}} \frac{d}{d \xi} \xi^{2} \frac{d}{d \xi} \vartheta=-\vartheta^{n},
$$

and the index "LE" is to remind the reader that the quantities are evaluated assuming that $\vartheta(\xi)$ is the LE function. $\mathcal{R}_{n, \ell}^{\mathrm{LE}}$ corresponds to Eq. (29) with $\vartheta(\xi)$ replaced by $\vartheta_{\mathrm{LE}}(\xi)$.

Reference [36] showed that when one applies all of the three conditions, $\bar{A}_{n, \ell}$ depends on the EOS, here parametrized through $n$, by at most $\mathcal{O}(10 \%)$ for $\ell \leq 4$ with $n=[0.3,1]$. This implies that the universal relations among lower $\ell$ multipoles depend only weakly on the EOS.
Moreover, through this analysis one finds that Eq. (34) is spin-independent.

The $\bar{I}-\bar{Q}$ relation discussed in the previous section can be reproduced as follows. First, using the self-similar isodensity condition [condition (1)] and Eq. (23), one finds

$$
\frac{\bar{M}_{2}}{\bar{I}}=-\frac{3 I_{2,3}}{2 \delta I_{1} \chi^{2}} .
$$

If one further imposes the elliptical condition [condition (2)], one finds [36]

$$
\frac{\bar{M}_{2}}{\bar{I}}=\frac{e^{2}}{2 \chi^{2}} .
$$

In order to see the EOS universality of the $\bar{I}-\bar{Q}$ relation for a fixed $\chi$, one needs to express $e$ in terms of $\chi, n$ and $\bar{I}$. Such an expression is given in Ref. [36] in the elliptical isodensity approximation, using the relation between $\Omega$ and $e$ found in Ref. [54].

In the remainder of this section, however, we will focus on how the universal relation among multipole moments is affected by relaxing the elliptical isodensity approximation. Reference [36] showed that the $\bar{I}-\bar{Q}$ relation has a similar (but slightly smaller) EOS variation to the $\bar{S}_{3}-\bar{M}_{4}$ relation, with the EOS dependence of the latter encoded in $\bar{A}_{n, 1}$. We thus expect that the $\bar{I}-\bar{Q}$ relations will be affected by the relaxation of the elliptical isodensity approximation in roughly the same way as the universal relation among multipole moments is affected. We will study the latter in detail in the next subsections, but we leave a detailed analysis of how the $\bar{I}-\bar{Q}$ relations are affected by the elliptical isodensity approximation to future work.

\section{B. Relaxing the elliptical condition}

Let us now relax each of the conditions made above in turn to see how it affects the universality. We start off by breaking the elliptical condition, since this is easiest. We can achieve this by choosing the function $\Theta(\mu)$ to be something other than Eq. (26). This function specifies the shape of the self-similar, isodensity contours. One could, for example, choose

$$
\Theta(\mu)=\left[\frac{1-e^{2}}{1-e^{2}\left(1-\mu^{2}\right)}\right]^{p / 2},
$$

for $p$ an odd, positive integer, i.e. $p \in \mathbb{N}_{\text {odd }}$. When $p=1$, one recovers an elliptical coordinate system, while for $p>$ 1 the star becomes more peanut-shaped (in the $\tilde{z}-\tilde{x}$ plane), and for $p<1$ it becomes more spherical, as shown in Fig. 12. Notice that when $e=0$, the star is spherical irrespective of the choice of $p$, since then $\Theta=1$. Interestingly, as $p$ increases, the peanut-shape becomes more and more pronounced. Clearly then, the cases when $p>1$ should be taken as toy problems only. In order to 


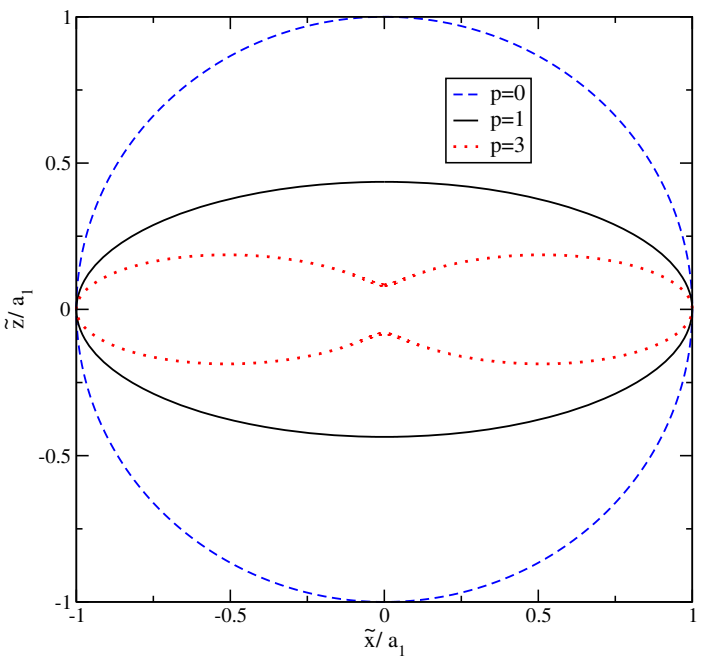

FIG. 12 (color online). Stellar shape in the $\tilde{x}-\tilde{z}$ plane given by Eq. (39) for different values of $p$ and $e=0.9$.

keep the analysis generic, we do not specify the form of $\Theta$ in this subsection.

Modifying the functional form of $\Theta$ only changes the angular integral $I_{\ell, k}$ in the multipole moment expression in Eq. (23). Thus, the relations among multipoles are still valid if they are expressed in terms of $I_{\ell, k}$, without explicitly solving $I_{\ell, k}$. It follows that the universal relations in Eqs. (25) and (31) with Eq. (32) still hold even if one relaxes the elliptical condition.

The first universal relation is automatically EOS independent, so let us focus on the EOS dependence of the second one. Let us consider the fractional difference of the barred coefficients from some mean $\left\langle\bar{A}_{n, \ell}\right\rangle$ on $n$. Since the EOS dependence is encoded in $\tilde{\mathcal{R}}_{n, \ell}$ of Eq. (32), one easily finds

$$
\frac{\bar{A}_{n, \ell}-\left\langle\bar{A}_{n, \ell}\right\rangle}{\left\langle\bar{A}_{n, \ell}\right\rangle}=\frac{\tilde{\mathcal{R}}_{n, \ell}-\left\langle\tilde{\mathcal{R}}_{n, \ell}\right\rangle}{\left\langle\tilde{\mathcal{R}}_{n, \ell}\right\rangle} .
$$

Since $\left\langle\tilde{\mathcal{R}}_{n, \ell}\right\rangle$ does not depend on $\Theta$, the fractional difference of $\bar{A}_{n, \ell}$ is the same even if one relaxes the elliptical condition. This shows that the shape of the self-similar surfaces does not affect the universality at all.

\section{Relaxing the spherical density profile condition}

Let us now consider the relaxation of the spherical density profile condition, namely the assumption that $\vartheta(\xi)$ is a LE function. One could, for example, let

$$
\vartheta(\xi)=\vartheta_{\mathrm{LE}}(\xi)\left[1+\epsilon_{1} \vartheta_{1}(\xi)\right],
$$

where $\epsilon_{1} \ll 1$ is some small number and $\epsilon_{1} \vartheta_{1}(\xi)$ is a small deformation. Such a modification to the isodensity condition can be easily propagated throughout the calculation of [36] described in Sec. III A, since effectively it reduces to the same integrals but with different harmonic number. The angular integrals, of course, are completely unmodified. The radial integrals can still be rescaled as in Eq. (28), except with the replacement

$$
\begin{aligned}
\mathcal{R}_{n, \ell} & \rightarrow \int_{0}^{\xi_{1}}[\vartheta(\xi)]^{n}\left[1+\epsilon_{1} \vartheta_{1}(\xi)\right]^{n} \xi^{\ell+2} d \xi, \\
& =\mathcal{R}_{n, \ell}+\epsilon_{1} n \mathcal{R}_{n, \ell}^{\left(\vartheta_{1}\right)}+\mathcal{O}\left(\epsilon_{1}^{2}\right),
\end{aligned}
$$

where we have expanded in $\epsilon_{1} \ll 1$ and

$$
\mathcal{R}_{n, \ell}^{\left(\vartheta_{1}\right)} \equiv \int_{0}^{\xi_{1}}[\vartheta(\xi)]^{n} \vartheta_{1}(\xi) \xi^{\ell+2} d \xi
$$

We see then that the modification to the density profile has changed the radial integral through the addition of a new term that depends on $\vartheta_{1}$ and $\epsilon_{1}$, but also on $n$ and $\ell$.

Let us now consider how these modifications affect the two universal relations described in Sec. III A. First, it is clear that the first relation is not modified at all since Eq. (25) does not depend on the radial integral. On the other hand, the second relation, given by Eq. (31), is modified since it depends on the radial integral through $\tilde{R}_{n, \ell}$ in Eq. (33). Using Eq. (42), $\bar{A}_{n, \ell}$ becomes

$$
\begin{aligned}
\bar{A}_{n, \ell}= & \frac{(2 \ell+3)^{1 / \ell}}{3^{(1+1 / \ell)}} \tilde{\mathcal{R}}_{n, \ell}^{\mathrm{LE}} \\
& \times\left\{1+\epsilon_{1} n\left[\frac{(\ell+1)}{\ell} \frac{\mathcal{R}_{n, 2}^{\left(\vartheta_{1}\right)}}{\mathcal{R}_{n, 2}^{\mathrm{LE}}}-\frac{1}{\ell} \frac{\mathcal{R}_{n, 2 \ell+2}^{\left(\vartheta_{1}\right)}}{\mathcal{R}_{n, 2 \ell+2}^{\mathrm{LE}}}-\frac{\mathcal{R}_{n, 0}^{\left(\vartheta_{1}\right)}}{\mathcal{R}_{n, 0}^{\mathrm{LE}}}\right]\right\} \\
& +\mathcal{O}\left(\epsilon_{1}^{2}\right) .
\end{aligned}
$$

From this result, we can already derive some interesting conclusions. The deviation from the standard universal relations are both linear in $\epsilon_{1}$ and $n$. In fact, how much the relations deviate will simply depend on the magnitude of $\epsilon_{1}$, since $n=\mathcal{O}(1)$.

\section{Relaxing the self-similar isodensity condition}

Let us relax the self-similar isodensity condition. In particular, one may wish to force the contours to become more spherical as $\tilde{r}$ approaches the NS core, which is in fact what occurs physically inside NSs [73]. Therefore, we promote the eccentricity to be a function of the radial coordinate, i.e.

$$
e \rightarrow e(\tilde{r})=e_{0} f\left(\frac{\tilde{r}}{a_{1}}\right)
$$

where $e_{0}$ is the eccentricity at the surface and $f$ is an arbitrary function of $\tilde{r} / a_{1}$ with $f(1)=1$. Although the relaxation of the self-similar isodensity condition through Eq. (45) renders the integrals of the previous subsections 
nonseparable in general, one can still perform the integrals analytically by calculating them as follows.

When the eccentricity becomes a function of the radial coordinate, $\Theta(\mu)$ in Eq. (26) now becomes $\Theta(\tilde{r}, \mu)$. In spite of this, one can still solve the angular integral exactly. The mass and spin moments then become

$$
\begin{aligned}
M_{\ell} & =2 \pi \int_{0}^{a_{1}} \rho(\tilde{r}) \tilde{r}^{\ell+2} I_{\ell, 3}(\tilde{r}) d \tilde{r}, \\
S_{\ell} & =\frac{4 \pi \ell}{2 \ell+1} \Omega \int_{0}^{a_{1}} \rho(\tilde{r}) \tilde{r}^{\ell+3} \delta I_{\ell}(\tilde{r}) d \tilde{r},
\end{aligned}
$$

where [36]

$$
\begin{aligned}
& I_{\ell, 3}(\tilde{r})=(-)^{\frac{\ell}{2}} \frac{2}{\ell+1} \sqrt{1-e(\tilde{r})^{2}} e(\tilde{r})^{\ell}, \\
& \delta I_{\ell}(\tilde{r})=(-)^{\frac{\ell-1}{2}} \frac{2(2 \ell+1)}{\ell(\ell+2)} \sqrt{1-e(\tilde{r})^{2}} e(\tilde{r})^{\ell-1} .
\end{aligned}
$$

One can rewrite Eqs. (46) and (47) as

$$
M_{\ell}=2 \pi I_{\ell, 3}^{(0)} R_{\ell}^{(M)}, \quad S_{\ell}=\frac{4 \pi \ell}{2 \ell+1} \Omega \delta I_{\ell}^{(0)} R_{\ell+1}^{(S)},
$$

where the superscript (0) refers to setting eccentricity constant [i.e. $e \rightarrow e_{0}=$ const in Eqs. (48) and (49)] and

$R_{\ell}^{(A)} \equiv \int_{0}^{a_{1}} \rho(\tilde{r}) \tilde{r}^{\ell+2}\left[f\left(\frac{\tilde{r}}{a_{1}}\right)\right] \sqrt[n_{A}]{\frac{1-e_{0}^{2}\left[f\left(\tilde{r} / a_{1}\right)\right]^{2}}{1-e_{0}^{2}}} d \tilde{r}$

with $A=(M, S), n_{M}=\ell$ and $n_{S}=\ell-2$. Observe the resemblance of Eqs. (50) and (23). Observe also that $R_{\ell}^{(M)}=R_{\ell}=R_{\ell}^{(S)}$ when $f\left(\tilde{r} / a_{1}\right)=1$.

\section{Modification to the Multipole Moments Relation}

Let us now investigate how the universal relations change by the replacement in Eq. (45). First, similar to Eq. (28), we define $\mathcal{R}_{n, \ell}^{(A)}$ as

$$
R_{\ell}^{(A)}=\rho_{c}\left(\frac{a_{1}}{\xi_{1}}\right)^{\ell+3} \mathcal{R}_{n, \ell}^{(A)},
$$

with

$\mathcal{R}_{n, \ell}^{(A)} \equiv \int_{0}^{\xi_{1}} \vartheta^{n} \xi^{\ell+2}\left[f\left(\frac{\xi}{\xi_{1}}\right)\right] \sqrt[n_{A}]{\frac{1-e_{0}^{2}\left[f\left(\xi / \xi_{1}\right)\right]^{2}}{1-e_{0}^{2}}} d \xi$.

We will here assume that $\vartheta$ is a LE function, but omit the index "LE" for convenience. In the slow-rotation limit, the above equation becomes

$$
\mathcal{R}_{n, \ell}^{(A)}=\left\{\int_{0}^{\xi_{1}} \vartheta^{n} \xi^{\ell+2}\left[f\left(\frac{\xi}{\xi_{1}}\right)\right]^{n_{A}} d \xi\right\}\left[1+\mathcal{O}\left(e_{0}^{2}\right)\right] .
$$

The first universal relation is obtained by substituting Eq. (52) into Eq. (50) and taking the ratio of these moments. After nondimensionalizing them through Eqs. (21) and (22), we find

$$
\frac{\bar{M}_{2 \ell+2}}{\bar{S}_{2 \ell+1}}=\bar{B}_{n, \ell}^{(f)} \bar{M}_{2}
$$

where we have defined

$$
\bar{B}_{n, \ell}^{(f)}=\frac{\mathcal{R}_{n, 2 \ell+2}^{(M)} \mathcal{R}_{n, 2}^{(S)}}{\mathcal{R}_{n, 2 \ell+2}^{(S)} \mathcal{R}_{n, 2}^{(M)}} .
$$

Notice that when $f\left(\tilde{r} / a_{1}\right)=1, \bar{B}_{n, \ell}^{(f)}=1$ and thus, one recovers the first universal relation of Eq. (27). Notice also that $\bar{B}_{n, 0}^{(f)}=1$ irrespective of the functional form of $f\left(\tilde{r} / a_{1}\right)$.

We follow the same procedure explained in Sec. III A to derive the second relation. Namely, we first rewrite $\rho_{c}$ in terms of $a_{1}$ and $M$ from Eqs. (50) and (52) with $\ell=0$, as in Eq. (30), with the radial integral performed using the LE equation. Next, we express $a_{1} / \xi_{1}$ in terms of $S_{2 \ell+1} / S_{1}$ from Eqs. (50) and (52). Then, from these equations, one finds

$$
\bar{M}_{2 \ell+2}=\bar{A}_{n, \ell}^{(f)}\left(\bar{S}_{2 \ell+1}\right)^{1+1 / \ell},
$$

where we have defined

$$
\bar{A}_{n, \ell}^{(f)}=\frac{(3+2 \ell)^{1 / \ell}}{3^{1+1 / \ell}} \frac{\mathcal{R}_{n, 2 \ell+2}^{(M)}\left(\mathcal{R}_{n, 2}^{(S)}\right)^{1+1 / \ell}}{\mathcal{R}_{n, 0}^{(M)}\left(\mathcal{R}_{n, 2 \ell+2}^{(S)}\right)^{1+1 / \ell}} .
$$

Notice again that when $f\left(\tilde{r} / a_{1}\right)=1$ one recovers the results of [36].

\section{Effect on universality}

We see clearly that the introduction of a nontrivial radial dependence in the eccentricity has led to different universal relations, but the question is whether such a modification spoils universality. We address this by looking at some example below. Let us consider the simple toy model

$$
f\left(\frac{\tilde{r}}{a_{1}}\right)=\left(\frac{\tilde{r}}{a_{1}}\right)^{s},
$$

for some power $s>0$ that controls how fast the star becomes spherical as one approaches the core. As shown in Fig. 13, when $s=0.3$, the star is mostly elliptical until $\tilde{r} / a_{1}<0.1$, at which point $e(\tilde{r})<e_{0} / 2$. On the other hand, when $s=3$ the star is more spherical, with its eccentricity dropping below half its initial value already at $\tilde{r} / a_{1}=0.8$. The actual $e(\tilde{r})$ for Newtonian stars can be estimated by 


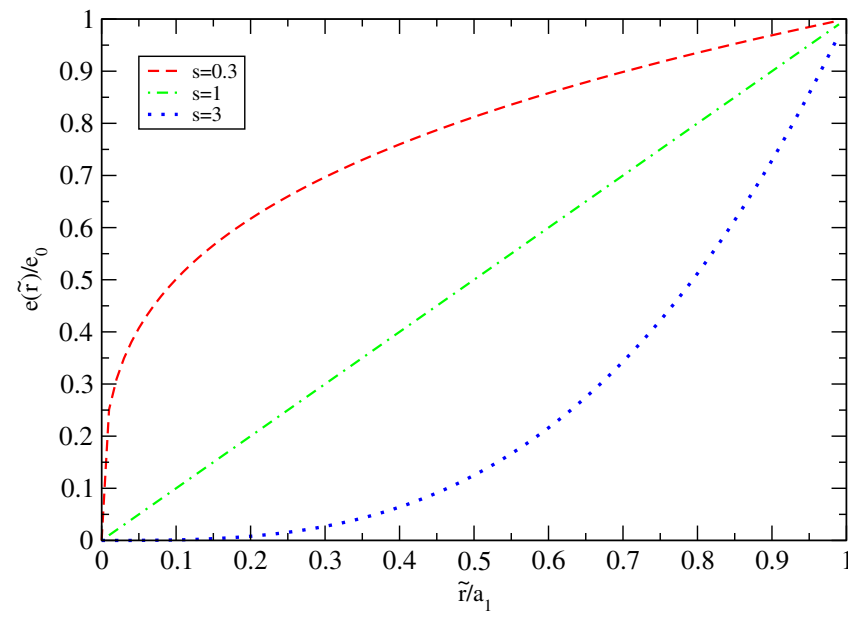

FIG. 13 (color online). Eccentricity as a function of radius given by Eq. (58) for different choices of $s$.

solving the Clairaut-Radau equation [73-75], as we will do in Sec. IV C. Since the purpose of this subsection is to investigate how $e(\tilde{r})$ affects the universality, we do not solve such equation but treat $s$ as arbitrary.

For simplicity, let us work in the slow-rotation limit. The coefficients in the universal relations then become

$$
\begin{aligned}
\bar{B}_{n, \ell}^{(f)} & =\bar{B}_{n, \ell, s} \equiv \frac{\mathcal{R}_{n, 2}}{\mathcal{R}_{n, 2+2 s}} \frac{\mathcal{R}_{n, 2 \ell+2+2 s(\ell+1)}}{\mathcal{R}_{n, 2 \ell+2+2 s \ell}}, \\
\bar{A}_{n, \ell}^{(f)} & =\bar{A}_{n, \ell, s} \\
& \equiv \frac{(3+2 \ell)^{1 / \ell}}{3^{1+1 / \ell}} \frac{1}{\xi_{1}^{2}\left|\vartheta_{1}^{\prime}\right|} \frac{\mathcal{R}_{n, 2 \ell+2+2 s(\ell+1)}\left(\mathcal{R}_{n, 2}\right)^{1+1 / \ell}}{\left(\mathcal{R}_{n, 2 \ell+2+2 s \ell}\right)^{1+1 / \ell}} .
\end{aligned}
$$

Figure 14 shows $\bar{B}_{n, \ell, s}$ (left panel) and $\bar{A}_{n, \ell, s}$ (right panel) as a function of $n$ for different choices of $s$ and $\ell$. Let us first focus on the first universal relation by looking at the left panel of Fig. 14. Recall that when $\ell=0, \bar{B}_{n, 0, s}=1$, which is why we plot only the $\ell \geq 1$ cases. Observe that irrespective of the choice of $\ell, \bar{B}_{n, \ell, 0.3} \sim \bar{B}_{n, \ell, 0}$, while $\bar{B}_{n, \ell, s}$ is very different from $\bar{B}_{n, \ell, 0}$ in the more spherical cases when $s>1$. Observe also that when $s=3$, the universality is essentially lost, with $\bar{B}_{n, \ell, s}$ changing by as much as $50 \%$ over the range of $n$ explored here. This is to be compared to a variability of about $1 \%$ when $s=0$ or 0.3 . Let us now look at the second universal relation by focusing on the right panel of Fig. 14. As in the case of $\bar{B}_{n, \ell, s}, \bar{A}_{n, \ell, s}$ is very similar when $s=0$ and when $s=0.3$. Also as before, the universality with $n$ deteriorates as $s$ increases, but the effect is much less pronounced this time.

In order to understand the behavior of $\bar{B}_{n, \ell}$, let us consider its asymptotic behavior in the $s \rightarrow \infty$ limit for an $n=0$ polytrope, where $\vartheta=1-\xi^{2} / 6$ and $\xi_{1}=\sqrt{6}$ so that $\mathcal{R}_{0, \ell}=6^{(\ell+3) / 2} /(\ell+3)$. First, the mass and current multipole moments in the slow-rotation limit become

$M_{2 \ell+2}=(-)^{\ell+1} \frac{4 \pi e_{0}^{2 \ell+2}}{2 \ell+3}\left(\frac{a_{1}}{\xi_{1}}\right)^{2 \ell+5} \frac{\rho_{c}}{\xi_{1}^{2 s(\ell+1)}} \mathcal{R}_{0,2 \ell+2+2 s(\ell+1)}$,

$S_{2 \ell+1}=(-)^{\ell} \frac{8 \pi \Omega}{2 \ell+3} e_{0}^{2 \ell}\left(\frac{a_{1}}{\xi_{1}}\right)^{2 \ell+5} \frac{\rho_{c}}{\xi_{1}^{2 s \ell}} \mathcal{R}_{0,2 \ell+2+2 s \ell}$

Then, from these equations, one finds

$$
M_{2 \ell+2} \sim \frac{1}{s}(\ell \geq 0), \quad S_{2 \ell+1} \sim \frac{1}{s}(\ell \geq 1),
$$

and $M \sim s^{0} \sim S_{1}$. Notice that both $M_{2 \ell+2}(\ell \geq 0)$ and $S_{2 \ell+1}(\ell \geq 1)$ vanish in the $s \rightarrow \infty$ limit. This is because in this limit, the density contour is spherical everywhere

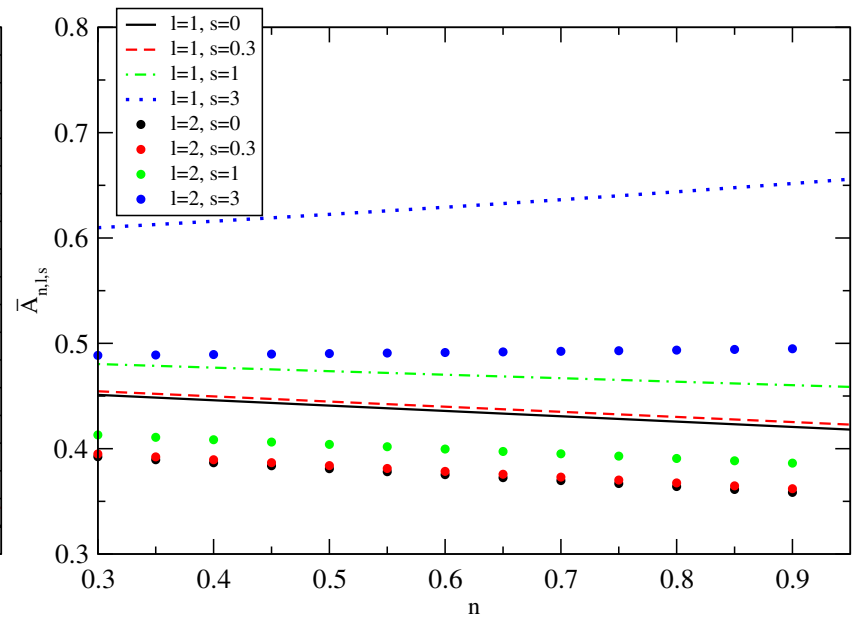

FIG. 14 (color online). $\quad \bar{B}_{n, \ell, s}$ (left) and $\bar{A}_{n, \ell, s}$ (right) given by Eqs. (59) and (60), respectively, as a function of $n$ for different choices of $s$ and $\ell$. Observe that as we increase $s$, the universality is lost, especially as $s>1$. Observe also that $\bar{B}_{n, \ell, s}$ and $\bar{A}_{n, \ell, s}$ are barely modified from the $s=0$ case when $s=0.3$. 
except at the surface. Since the measure of the integrals is zero, the multipole moments vanish. The above scaling leads to

$$
\bar{B}_{0, \ell}=\frac{M_{2 \ell+1} S_{1}}{S_{2 \ell+1} M_{2}} \sim s
$$

in the large $s$ limit. For $n$ close to zero, $\bar{B}_{n, \ell}$ can be expressed as $\bar{B}_{n, \ell} \sim\left(c_{0}+c_{1} n\right) s$ for some constants $c_{0}$ and $c_{1}$ and the fractional difference of $\bar{B}_{n, \ell}$ with respect to some mean $n$ is given by

$$
\frac{\bar{B}_{n, \ell}-\left\langle\bar{B}_{n, \ell}\right\rangle}{\left\langle\bar{B}_{n, \ell}\right\rangle} \sim \frac{c_{1}(n-\langle n\rangle)}{c_{0}+c_{1}\langle n\rangle} .
$$

Therefore, for large $s$, the fractional difference of $\bar{B}_{n, \ell}$ is nonvanishing and can be relatively large.

\section{ECCENTRICITY PROFILES FOR RELATIVISTIC STARS}

The previous section showed that the elliptical isodensity approximation plays a crucial role in the universality. We now need to investigate the validity of such an approximation for realistic relativistic stars, i.e. study the eccentricity radial profile of such stars, thus addressing the third question in the Introduction.

We will first look at the profile of slowly rotating NSs and QSs. We will show that the eccentricity only changes by $\sim 10 \%$ within the region that matters to the universality. We will then look at the profile of rapidly rotating NSs and show that the eccentricity variation is always smaller than $20 \%-30 \%$ even for rapidly rotating NSs in the region that matters. We will finally re-consider the relation for uniformly rotating Newtonian polytropes described in Sec. III D using a realistic eccentricity profile for NSs. We will show that such modification only affects the 3-hair relation in [36] by less than $10 \%$ relative to the constanteccentricity Newtonian results, e.g. if certain relations are universal to $1 \%$ when using the elliptical isodensity approximation, corrections due to a nonconstant eccentricity profile induce modifications of $0.1 \%$.

\section{A. Slowly rotating stars}

We first look at eccentricity profiles for slowly rotating NSs and QSs. We extract the eccentricity of isodensity surfaces from the embedded surface following [56]. Figure 2 shows the radial profile of the NS eccentricity, which affects the stellar quadrupole moment. Within $50 \%-95 \%$ of the stellar radius, the eccentricity only changes by $\sim 10 \%$. Observe also that the EOS variation in this radial region is smaller than the one in the region less than $50 \%$ of the radius.

Figure 15 shows the eccentricity profile for QSs. Observe that the eccentricity is almost constant for QSs with large $\bar{I}$. For such stars, the elliptical isodensity

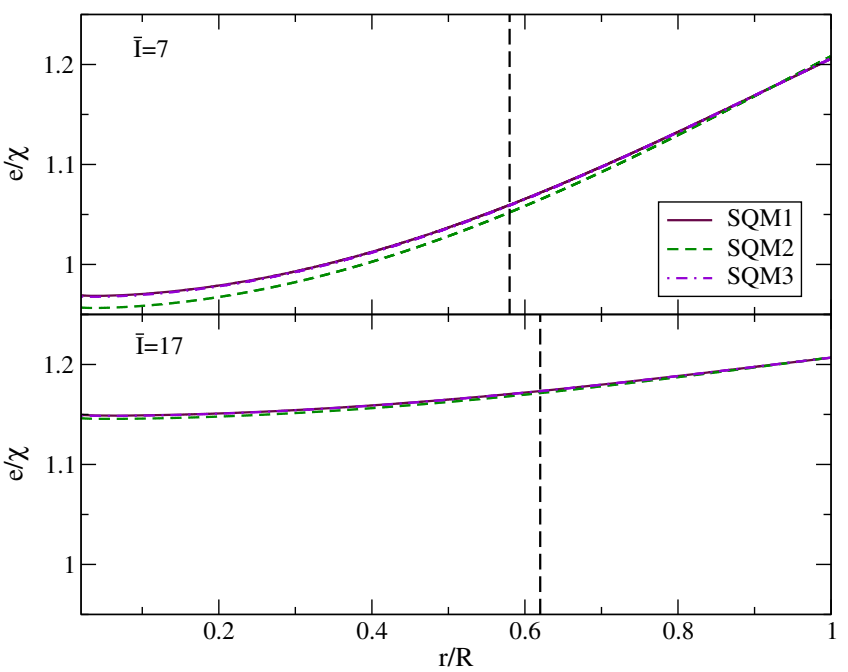

FIG. 15 (color online). Eccentricity profile (same as Fig. 2) but for QSs. The vertical dashed lines are built in the same way as in Fig. 8. Observe that the eccentricity does not change much for stars with large $\bar{I}$.

approximation used in [36] becomes an excellent approximation. Such approximate self-similarity in the stellar eccentricity suppresses the EOS variation in the relations among stellar multipole moments, which in turn realizes the universality.

\section{B. Rapidly rotating stars}

Let us now study the eccentricity radial profile for rapidly rotating stars. As in the slow-rotation case, we estimate the eccentricity of constant density surfaces from the embedded surface following [56]. We will present the results in Schwarzschild-like coordinates. Figure 16 presents the NS eccentricity profile for $\bar{n}=0.5$ (top) and $\bar{n}=1$ (bottom) polytropic indices, for the various rotation rates of Fig. 11. Observe that in the Newtonian limit (left panels), the Clairaut-Legendre approximation ${ }^{3}$ (red curves) gives an accurate description of the radial profile of the eccentricity for all the rotating models (green curves). This approximation becomes less accurate as the compactness increases (right panels). Observe that the eccentricity variation due to rotation becomes larger as one increases $\lambda$ and rotation. The relativistic effects with a rapid rotation result in lower central values for the eccentricity and thus higher eccentricity variation throughout the star. The observed behavior of the relativistic eccentricity profile is consistent with the Newtonian picture [73], where the more centrally condensed polytropes have larger variation in their eccentricity profiles, in agreement with previous results [76].

\footnotetext{
${ }^{3}$ The Clairaut-Legendre approximation refers to the expansion calculated by Clairaut using Legendre polynomials [73], not to be confused with the Clairaut-Radau equation.
} 

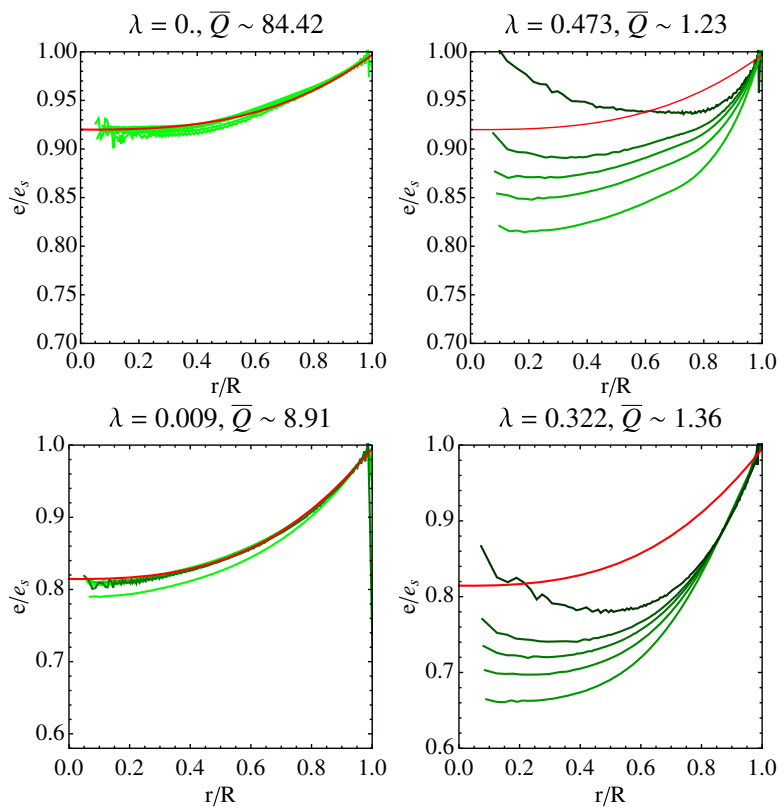

FIG. 16 (color online). Eccentricity radial profile (normalized by the eccentricity at the stellar surface $e_{s}$ ) with a polytropic index $\bar{n}=0.5$ (top) and $\bar{n}=1$ (bottom). The red curve shows the Newtonian slow-rotation, Clairaut-Legendre model, while the five green curves correspond to the same relativistic rotating models as in Fig. 11. The strange behavior of the eccentricity of the central regions in the right panels is mainly due to the specific coordinates used to measure the eccentricity, which have no specific analogue in Newtonian stars.

Although the relativistic effects lower the central value of the eccentricity compared to the corresponding Newtonian value, the actual variation does not exceed $35 \%$, while the variation is $\sim 20 \%$ throughout a Newtonian $\bar{n}=1$ polytrope. In the region that matters to the universality $(r / R=0.5-0.95)$, the eccentricity varies by $20 \%-30 \%$, validating the elliptical isodensity approximation to $\mathcal{O}(10 \%)$ even for rapidly rotating NSs.

\section{Newtonian multipole relations with realistic eccentricity profile}

Let us now consider the Newtonian analysis described in Sec. III D but using a realistic eccentricity profile. One sees from Figs. 15 and 16 that the eccentricity for realistic stellar models remains nonvanishing at the stellar center. Therefore, one can promote the eccentricity profile to

$$
f\left(\frac{\tilde{r}}{a_{1}}\right)=1+\delta e\left[\left(\frac{\tilde{r}}{a_{1}}\right)^{s}-1\right]
$$

where $\delta e$ and $s$ are constants. One can obtain $\delta e$ and $s$ for the slowly rotating Newtonian polytropes by solving the Clairaut-Radau equation [73-75], as shown in Fig. 17.

One can use these realistic $\delta e$ and $s$ as functions of $n$ to calculate the coefficients $\bar{B}_{n, \ell}^{(f)}$ and $\bar{A}_{n, \ell}^{(f)}$ in the universal

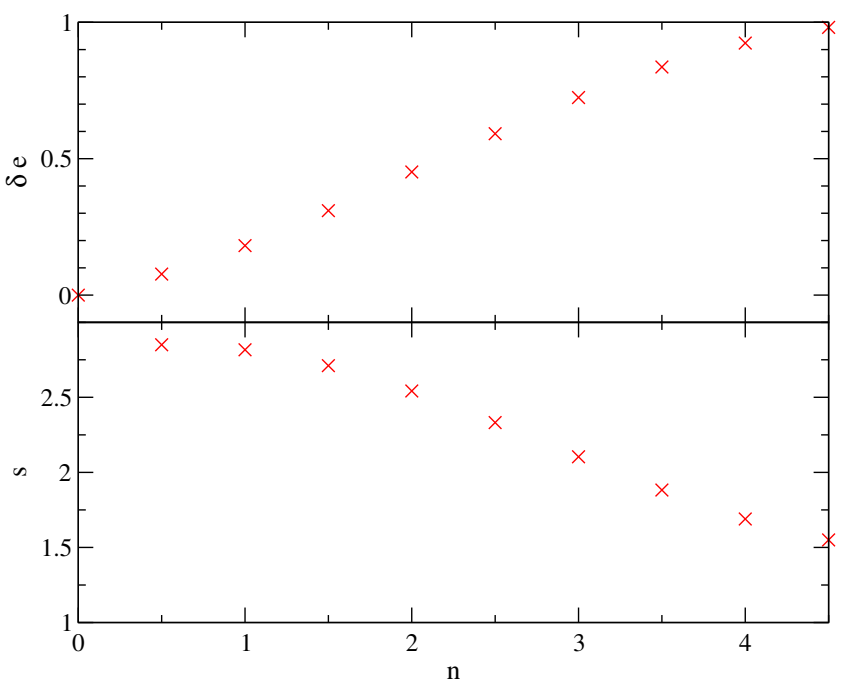

FIG. 17 (color online). $\quad \delta e$ and $s$ in Eq. (66) as functions of $n$ for Newtonian polytropes.

relations. Figure 18 shows the fractional difference of $\bar{B}_{n, \ell}^{(f)}$ and $\bar{A}_{n, \ell}^{(f)}$ from those with $f\left(\tilde{r} / a_{1}\right)=1$ (or equivalently, $\delta e=0$ ) as functions of $n$ for various $\ell$ in the slow-rotation limit. Notice that the fractional difference decreases as one decreases $n$. This is because the elliptical isodensity approximation becomes exact for an $n=0$ polytrope. Notice also that this fractional difference is not a measure of universality, but rather it is a measure of how sensitive the universal relations are to the nonconstant eccentricity profile. For example, if in the constant eccentricity case, the relation between the multipoles were accurate to $1 \%$, then the fractional difference of Fig. 18 shows how a nonconstant eccentricity profile affects this $1 \%$ accuracy by $\sim 0.1 \%$ at most.

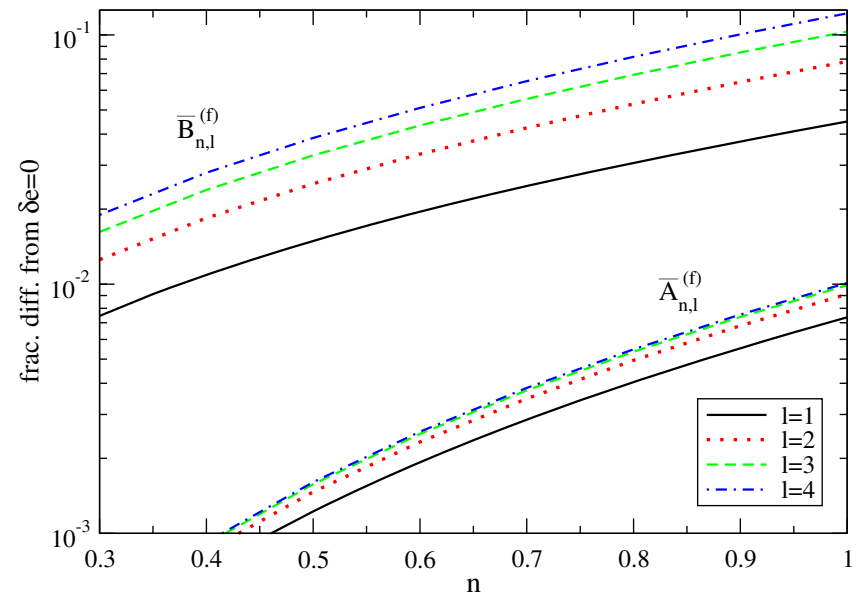

FIG. 18 (color online). Fractional difference of $\bar{B}_{n, \ell}^{(f)}$ [Eq. (55)] and $\bar{A}_{n, \ell}^{(f)}$ [Eq. (57)] based on Eq. (66) and Fig. 17 from those with constant eccentricity $(\delta e=0)$ as functions of $n$ in the slowrotation limit. 
Figure 18 shows that the fractional difference of $\bar{B}_{n, \ell}^{(f)}$ is larger than that of $\bar{A}_{n, \ell}^{(f)}$. One can understand such behavior by expanding the universal relations around $\delta e=0$. To do so, one first needs to expand $\mathcal{R}_{n, \ell}^{(M)}$ and $\mathcal{R}_{n, \ell}^{(S)}$ to yield

$\mathcal{R}_{n, \ell}^{(M)}=\mathcal{R}_{n, \ell}\left[1+\frac{2 \ell-(2 \ell+1) e_{0}^{2}}{2\left(1-e_{0}^{2}\right)} \delta e \frac{\mathcal{R}_{n, \ell, s}}{\mathcal{R}_{n, \ell}}\right]+\mathcal{O}\left(\delta e^{2}\right)$,

$$
\begin{aligned}
\mathcal{R}_{n, \ell}^{(S)}= & \mathcal{R}_{n, \ell}\left[1+\frac{2(\ell-2)-(2 \ell-3) e_{0}^{2}}{2\left(1-e_{0}^{2}\right)} \delta e \frac{\mathcal{R}_{n, \ell, s}}{\mathcal{R}_{n, \ell}}\right] \\
& +\mathcal{O}\left(\delta e^{2}\right),
\end{aligned}
$$

where

$$
\mathcal{R}_{n, \ell, s} \equiv \int_{0}^{\xi_{1}} \vartheta^{n} \xi^{\ell+2}\left[\left(\frac{\xi}{\xi_{1}}\right)^{s}-1\right] d \xi
$$

Substituting Eqs. (67) and (68) into Eqs. (55) and (57), one finds

$$
\begin{aligned}
\bar{B}_{n, \ell}^{(f)}= & 1+2 \delta e\left(\frac{\mathcal{R}_{n, 2 \ell+2, s}}{\mathcal{R}_{n, 2 \ell+2}}-\frac{\mathcal{R}_{n, 2, s}}{\mathcal{R}_{n, 2}}\right)+\mathcal{O}\left(\delta e^{2}\right), \\
\bar{A}_{n, \ell}^{(f)}= & \bar{A}_{n, \ell}\left[1+\frac{e_{0}^{2} \delta e}{2\left(1-e_{0}^{2}\right)}\left(\frac{1}{\ell} \frac{\mathcal{R}_{n, 2 \ell+2, s}}{\mathcal{R}_{n, 2 \ell+2}}-\frac{\ell+1}{\ell} \frac{\mathcal{R}_{n, 2, s}}{\mathcal{R}_{n, 2}}\right.\right. \\
& \left.\left.+\frac{\mathcal{R}_{n, 0, s}}{\mathcal{R}_{n, 0}}\right)\right]+\mathcal{O}\left(\delta e^{2}\right) .
\end{aligned}
$$

One now sees that the correction to $\bar{B}_{n, \ell}^{(f)}$ is $\mathcal{O}(\delta e)$ while that to $\bar{A}_{n, \ell}^{(f)}$ is $\mathcal{O}\left(\delta e e_{0}^{2}\right)$.

Figure 18 shows that the fractional difference increases as $\ell$ increases. This is somewhat surprising: for higher- $\ell$, the multipole moments are more heavily weighted toward the surface, precisely where the elliptical isodensity approximation should be more accurate, and thus, one expects the fractional difference to decrease as $\ell$ increases. Figure 18, however, shows the opposite behavior. The mass multipole moments are roughly given by $M_{\ell} \sim \int_{0}^{a_{1}}$ $\rho(\tilde{r}) \tilde{r}^{\ell+2} e(\tilde{r})^{\ell} d \tilde{r}$. Because of the $\tilde{r}^{\ell+2}$ factor in the integrand, it is true that the multipole moments have more weight on the outer region inside the star. However, notice that the integrand also contains a factor of $e^{\ell}$. Since the eccentricity is raised to the $\ell$ th power, the multipole moments are more sensitive to the difference between the realistic and constant eccentricity stellar models as $\ell$ increases. Therefore, whether the fractional difference in the universal relations increases as one increases $\ell$ depends on which of these two effects dominate.

In order to quantify the $\ell$ dependence in the fractional difference of the universal relations from the constant eccentricity case, we perform the following scaling estimate in the slow-rotation $\left(e_{0} \ll 1\right)$ and nearly constant eccentricity $(\delta e \ll 1)$ limit. Since all the eccentricity dependence in the universal relations is encoded in $\mathcal{R}_{n, \ell}^{(A)}$, let us focus on this quantity. Using the approximations described above, Eq. (67) leads to

$$
\mathcal{R}_{n, \ell}^{(M)}=\mathcal{R}_{n, \ell}\left[1+\ell \delta e \frac{\mathcal{R}_{n, \ell, s}}{\mathcal{R}_{n, \ell}}\right]+\mathcal{O}\left(\delta e^{2}, e_{0}^{2}\right) .
$$

The fractional difference from the constant eccentricity case is then given by

$$
\frac{\left|\mathcal{R}_{n, \ell}^{(M)}-\mathcal{R}_{n, \ell}\right|}{\mathcal{R}_{n, \ell}}=\ell \delta e \frac{\left|\mathcal{R}_{n, \ell, s}\right|}{\mathcal{R}_{n, \ell}} .
$$

As an example, let us use $n \sim 0$, and then

$$
\begin{aligned}
\mathcal{R}_{n, \ell, s} & \sim \int_{0}^{\xi_{1}} \xi^{\ell+2}\left[\left(\frac{\xi}{\xi_{1}}\right)^{s}-1\right] d \xi=-\frac{s \xi_{1}^{\ell+3}}{(\ell+3)(\ell+s+3)}, \\
\mathcal{R}_{n, \ell} & \sim \int_{0}^{\xi_{1}} \xi^{\ell+2} d \xi=\frac{\xi_{1}^{\ell+3}}{(\ell+3)},
\end{aligned}
$$

and

$$
\frac{\left|\mathcal{R}_{n, \ell}^{(M)}-\mathcal{R}_{n, \ell}\right|}{\mathcal{R}_{n, \ell}} \sim \delta e \frac{\ell s}{\ell+s+3},
$$

which monotonically increases from $0(\ell=0)$ to $s$ $(\ell=\infty)$. One can easily obtain a similar result for the fractional difference in $\mathcal{R}_{n, \ell}^{(S)}$. This rough estimate shows that the fractional difference of the universal relations from the constant eccentricity case increases as one increases $\ell$, as shown in Fig. 18. Since the figure shows that the correction from the constant eccentricity case to the universal relations are $\sim 10 \%$ at most for $\ell \leq 4$, this justifies the use of the elliptical isodensity approximation to model realistic stars.

\section{MULTIPOLE RELATIONS AND ECCENTRICITY PROFILE FOR NONCOMPACT STARS}

In order to better understand the relationships satisfied by the multipole moments of neutron stars, it is instructive to understand how they are different from regular, noncompact stars. We simulate regular rotating stars to address two main questions:

(i) Do regular stars satisfy an $\bar{I}-\bar{Q}$ relation or some other multipole moment relation?

(ii) Is the elliptical isodensity approximation valid for regular stars?

In this section, we address these questions, which are essentially those posed in the fourth item of the Introduction. The answers to these questions will provide further justification of the picture of an emergent symmetry as an explanation for the universality, already described in the Introduction and in more detail in the next section. 
WHY I-LOVE-Q: EXPLAINING WHY UNIVERSALITY ...

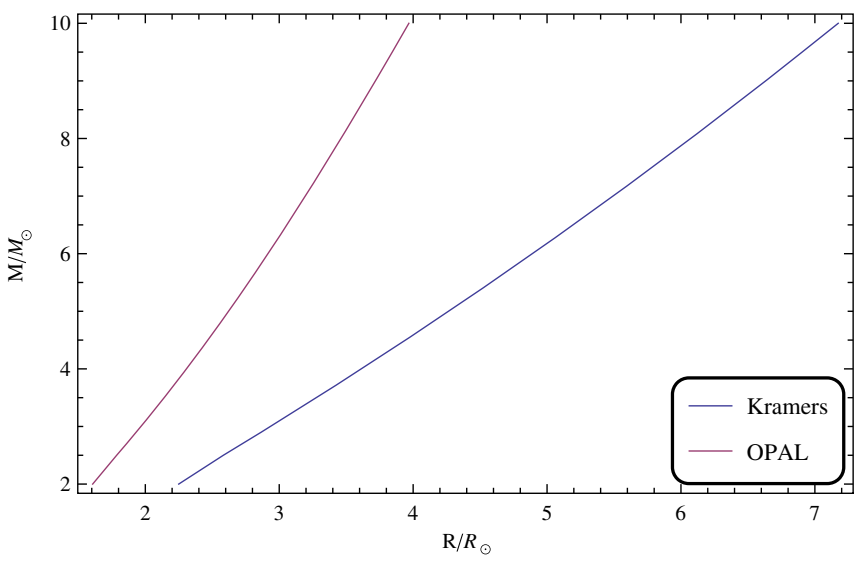

FIG. 19 (color online). The mass-radius relation of noncompact stars with two different opacity laws. Observe that different opacity laws corresponds to different effective EOSs.

We simulate a series of rapidly rotating stellar models, gridded in the mass range $M_{*} \in\left[2 M_{\odot}, 10 M_{\odot}\right]$ in increments of $0.5 M_{\odot}$ and in the range of surface equatorial rotation frequencies (as a fraction of breakup) $\Omega_{\mathrm{Eq}}^{\text {Surf }} \in$ $\left[0.1 \Omega_{\mathrm{bk}}, 0.9 \Omega_{\mathrm{bk}}\right]$ in increments of $0.1 \Omega_{\mathrm{bk}}$. Such rotation rates correspond to $\chi=3-17.5$ for a $3 M_{\odot}$ star. To perform these simulations we used the publicly-available ESTER code $[60,61]$, which self-consistently solves the equations of stellar structure for an axisymmetric, rapidly and differentially rotating star with realistic tabulated EOS. ${ }^{4}$ We cannot freely vary the equation of state, since it is well understood for such a star. However, we can vary the opacity law as a proxy to understand the EOS dependence. We adopted two different opacity laws: Kramers' law and the OPAL tabulated opacity [77]. The latter is more realistic, while we consider the former as a toy model to see the effective EOS universality for noncompact stars. Figure 19 shows the mass-radius relation for two different opacity laws. Observe that changing the opacity law has an effect on the mass-radius relation similar to changing the EOS. We address questions (i) and (ii) in Secs. VA and $\mathrm{VB}$, respectively.

\section{A. Multipole relationships}

As the ESTER code is spectral, it is straightforward to extract the multipole moments from the solutions. ${ }^{5}$ So far in this paper, we have used $I=S_{1} / \Omega$ as a definition for the moment of inertia. This definition is appropriate for rigid rotation, but here we are considering differentially-rotating stars. Therefore, in this section, we take advantage of the Newtonian limit and instead use

\footnotetext{
${ }^{4}$ ester-project: Evolution STEllaire en Rotation, available at https://code.google.com/p/ester-project/. Accessed April 2014.

${ }^{5}$ Program available at https://github.com/duetosymmetry/ester/ tree/master/src/multipoles/. Accessed April 2014.
}

PHYSICAL REVIEW D 90, 063010 (2014)

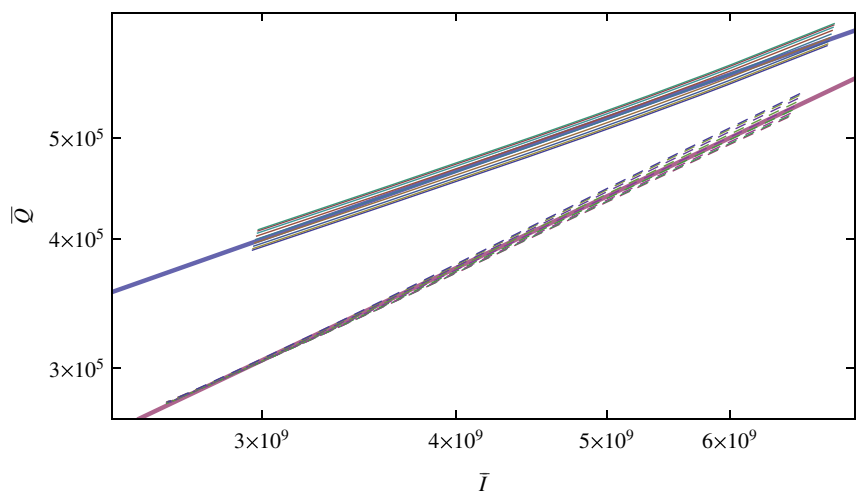

FIG. 20 (color online). $\quad \bar{I}-\bar{Q}$ relation for regular stars of various masses, spins, and opacity laws of Kramers' (solid) and OPAL (dashed). Within each opacity law family, each curve has a constant $\Omega_{\mathrm{Eq}}^{\text {Surf }} / \Omega_{\mathrm{bk}}=0.1,0.2, \ldots 0.9$. Along each curve, total stellar mass increases to the left, with $M_{*}=2 M_{\odot}$ at the rightmost end and $M_{*}=10 M_{\odot}$ at the leftmost end. Within each family there is $2 \%-3 \%$ variation from the best fit, while the two fits differ from each other by $40 \%$ at most.

$$
I^{N}=\frac{2}{3} \int \rho r^{2} d V
$$

where the integral is over the entire star; this is Eq. (9) without any symmetry assumptions.

The $\bar{I}-\bar{Q}$ relationship for regular stars is shown in Fig. 20 with Kramers' (solid) and OPAL (dashed) opacity laws. Each curve in each opacity family is at a constant $\Omega_{\mathrm{Eq}}^{\text {Surf }} / \Omega_{\mathrm{bk}}$, with $M_{*}$ varying along a curve (and increasing to the left). We create a fit for each opacity family given by a power-law of the form $\bar{Q}=C \bar{I}^{b_{1}}$ by fitting a straight line in $\log$ space, i.e.

$$
\log \bar{Q}=b_{1} \log \bar{I}+b_{2} .
$$

The fit parameters are presented in Table II. The fractional difference between these two fits can be as large as $40 \%$, showing a loss of universality. Notice that the dependence on $\Omega$ is weak, about $2 \%-3 \%$ variation, unlike in the relation for compact stars, which has a clear spin dependence [33-35,78].

Recall that in the Newtonian limit $[26,36]$, the $\bar{I}-\bar{Q}$ relation for compact stars is $\bar{Q} \propto \bar{I}^{1 / 2}$. From the $b_{1}$ parameter in Table II we see that noncompact stars lie

TABLE II. Fit parameters for the power-law parametric form in Eq. (77) for the two families with different opacity laws. Each family has an approximately $2 \%-3 \%$ variation from the fit, and the two fits have an approximately $40 \%$ variation at most from each other.

\begin{tabular}{lcc}
\hline \hline Opacity & $b_{1}$ & \multicolumn{1}{c}{$b_{2}$} \\
\hline Kramers & $0.527 \pm 0.0051$ & $-1.39 \pm 0.11$ \\
OPAL & $0.712 \pm 0.0022$ & $-2.90 \pm 0.050$ \\
\hline \hline
\end{tabular}




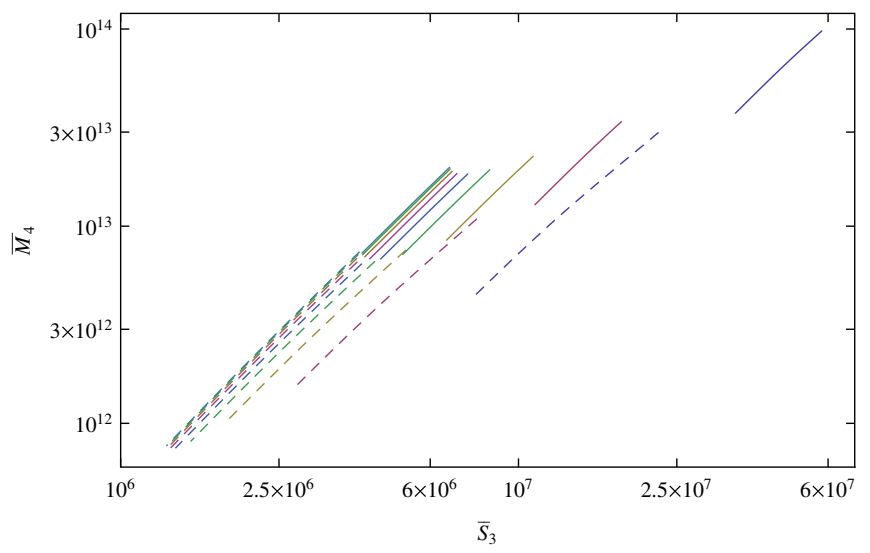

FIG. 21 (color online). The $\bar{S}_{3}-\bar{M}_{4}$ relation for regular stars with Kramers' (solid) and OPAL (dashed) opacity law, which fails to exhibit universality in both effective EOS (opacity law) and spin. Within each family, each curve is for a fixed $\Omega_{\mathrm{Eq}}^{\text {Surf }} / \Omega_{\mathrm{bk}}=0.1,0.2, \ldots 0.9$ which increases to the right amongst curves. Along each curve, mass increases to the left. Observe how the effective EOS universality is lost especially for a fixed, relatively large $\Omega_{\mathrm{Eq}}^{\text {Surf }} / \Omega_{\mathrm{bk}}$.

on different $\bar{I}-\bar{Q}$ relations than compact stars. We also see that changing the opacity law, which is our proxy for the EOS, changes the fit, and so noncompact stars do not possess an $\bar{I}-\bar{Q}$ relation that is nearly as universal as that which hold for compact stars.

Compact stars also enjoy additional relationships amongst higher multipole moments that are also approximately universal $[33,36]$; is such universality still present for noncompact stars? Figure 21 shows the relation between $\bar{S}_{3}$ and $\bar{M}_{4}$ for noncompact stars, which should be the next most-universal combination after $\bar{I}-\bar{Q}$ [36], for Kramers' (solid) and OPAL (dashed) opacity laws. Within each opacity family, we vary $\Omega_{\mathrm{Eq}}^{\text {Surf }} / \Omega_{\mathrm{bk}}$. For slowly rotating stars, the effective EOS universality is preserved, while it is lost for rapidly rotating stars with a fixed dimensionless spin parameter of $\Omega_{\mathrm{Eq}}^{\text {Surf }} / \Omega_{\mathrm{bk}}$. The figure also exhibits a clear spin dependence in the relations, which is in contrast to the relation for compact stars $[33,35]$. Such loss in the universality could be considered as a consequence of the breakdown of the elliptical isodensity approximation, as we will see in the next subsection.

\section{B. Isodensity contours}

We now turn to the question of the validity of the elliptical isodensity contour approximation for regular stars. Isodensity contours were extracted from the ESTER simulations by root-finding, ${ }^{6}$ and then computing the best-fit ellipse to each contour. Figure 22 shows an example of the radial dependence of $e / \chi$ with Kramers'

\footnotetext{
${ }^{6}$ Program available at https://github.com/duetosymmetry/ester/ tree/master/src/iso_contours. Accessed April 2014. This program makes use of the GNU Scientific Library [79].
}

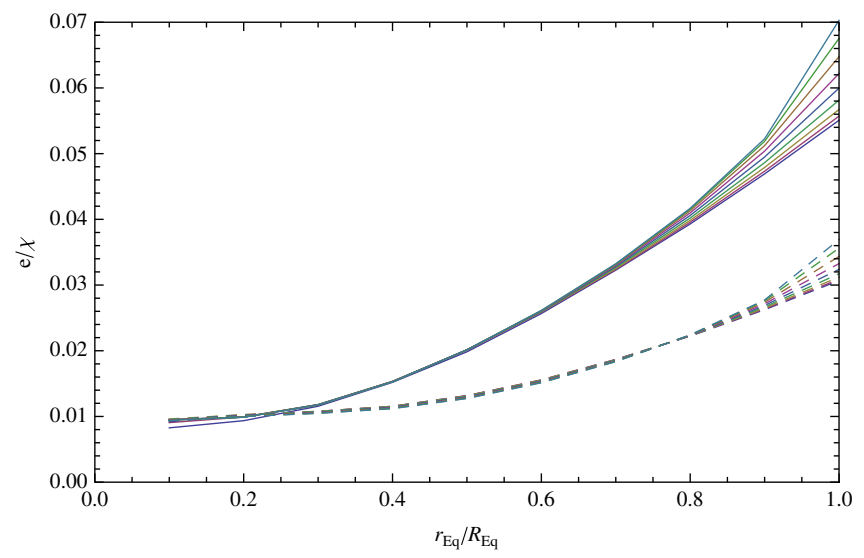

FIG. 22 (color online). Radial dependence of $e / \chi$ values in regular stars with Kramers' (solid) and OPAL (dashed) opacity law. $r_{\mathrm{Eq}}$ and $R_{\mathrm{Eq}}$ correspond to the radial coordinate in the equatorial plane and the stellar equatorial radius, respectively. The values are best-fits to isodensity contours. Within each family, each curve corresponds to one value of $\Omega_{\mathrm{Eq}}^{\text {Surf }} / \Omega_{\mathrm{bk}}=$ $0.1,0.2, \ldots 0.9$, increasing upwards. We set the mass as $M=5 M_{\odot}$, but the result presented in this figure is insensitive to the choice of $M$.

(solid) and OPAL (dashed) opacity laws with $M=5 M_{\odot}$ for different spin frequencies. The fits are quite faithful, with fractional errors between the contour and fit of at worst (i.e. at the stellar surface) $7 \%$, and sub- $1 \%$ for spins below $\Omega_{\mathrm{Eq}}^{\text {Surf }}<0.7 \Omega_{\mathrm{bk}}$.

From these fits we infer another important difference between compact and noncompact stars. In Figs. 2 and 15 we saw that the $e / \chi$ does not vary much in the range of radii that contribute the most to the moment of inertia and quadrupole moment. By comparison, for regular stars, we can see from Fig. 22 that $e / \chi$ varies by a factor of $\sim 3-6$ throughout the entire star, much more than for compact stars. Such large eccentricity variation is precisely the reason why the effective EOS universality is lost for the $\bar{I}-\bar{Q}$ and $\bar{S}_{3}-\bar{M}_{4}$ relations in general. These results provide further evidence for the picture of an emergent symmetry as the origin of universality, which we will explain in more detail in the next section.

\section{UNIVERSALITY AS AN EMERGENT APPROXIMATE SYMMETRY}

Let us now summarize our work and describe in detail the picture of emergent approximate symmetry that arises as an explanation for the universality.

In this paper, we provided further evidence of why the universal relations hold among stellar multipole moments. We first tackled this problem by looking at the $\bar{I}-\bar{Q}$ relation using piecewise polytropic EOSs [57] that can reproduce various realistic EOSs with just five free parameters. Although varying each piecewise parameter by $30 \%$ significantly modifies the mass-radius relation, we found 
that it only affects the $\bar{I}-\bar{Q}$ relation by $\mathcal{O}(1 \%)$ at most. We also found that the radial profile of the integrand of the moment of inertia and quadrupole moment is hardly affected by the piecewise parameters for a fixed moment of inertia, and most of the contribution in the moment of inertia and quadrupole moment comes from $50 \%-95 \%$ of the radius for NSs, confirming $[25,26]$. The parameters that control the density in the range $\left(10^{14}-10^{15}\right) \mathrm{g} / \mathrm{cm}^{3}$ play the most important role in the universality. The variation in the slope of the nuclear EOS within this region can be as large as $17 \%$ and we concluded that this alone cannot explain the universality.

Second, we extended the work in [36] in the Newtonian limit. We relaxed the elliptical isodensity approximation [54] to see how this assumption affects the universality. We found that the shape of the isodensity contours does not affect the universality, but relaxing the self-similarity condition does modify the relation significantly. This shows that the fact that isodensity contours of NSs can be approximated by self-similar surfaces plays a crucial role in universality.

Third, we investigated the eccentricity profile for both slowly and rapidly rotating relativistic stars. We showed that the eccentricity only varies by $\sim 10 \%$ in the region that matters to the universality for slowly-rotating NSs and QSs, while the eccentricity variation is below $20 \%-30 \%$ even for rapidly rotating NSs. This suggests the elliptical isodensity approximation is a very good description for realistic NSs. To verify this, we improved the Newtonian analysis in [36] by using a realistic, radially-dependent eccentricity profile for NSs. We found that, in the slow-rotation limit, the radial dependence of the eccentricity introduces at most a $10 \%$ correction to the $\bar{I}-\bar{Q}$ relation within the constant eccentricity assumption.

Fourth, we looked at the multipole relations and eccentricity profiles for noncompact, regular stars. We found that the eccentricity variation can easily exceed $100 \%$ for such stars, in contrast to relativistic stars that only have $\sim 10 \%$ variation for similar rotation rates. This means that the elliptical isodensity approximation is not good to model noncompact stars. We then studied whether the $\bar{I}-\bar{Q}$ relations remained universal for noncompact stars when we change its EOS. As a proxy for the latter, we used two different opacity laws, which indeed lead to different slopes in the mass-radius relation. We found that the $\bar{I}-\bar{Q}$ relations and the relations among higher multipole moments are not EOS universal for noncompact, regular stars.

The above results paint an interesting, albeit phenomenological picture of why universality holds. Consider the multi-dimensional phase space, spanned by different quantities that characterize stars, such as their stellar temperature, compactness, rotation rate, strength of the magnetic field, etc. One corner of this space is inhabited by very hot and noncompact stars, like supergiants, with stellar compactness of about $10^{-8}$ and temperatures of $20,000 \mathrm{~K}$, which may rotate differentially in some interior region. Since the interior density in such stars is subnuclear, the EOS can be well modeled through simulations, experimental data and helio-seismological observations. Another corner of this space is inhabited by cold (relative to their Fermi temperature) and compact stars, like NSs with compactness of about $10^{-1}$ and temperatures of $10^{-9} T_{F}$, where $T_{F}$ is the Fermi temperature. These stars rotate rigidly in the absence of external perturbations, because in the barotropic limit, vorticity (and thus differential rotation) is unsourced. Since the interior density in such stars is supra-nuclear, the EOS is not perfectly well-understood.

In spite of our ignorance of the NS's EOS, the corner of phase space in which they live is effectively dominated by just two dimensions: compactness and EOS effective polytropic index $n$. All other extra dimensions related to microphysics, like temperature, play a small, negligible role in controlling the characteristics of NSs. That is, at sufficiently high compactness, all details of microphysics efface away and NSs can be well-described by barotropic EOSs. Effectively, this EOS can be approximately captured by a polytrope, with an effective polytropic index $n \in$ $[0.3,1]$.

Let us then span this corner of phase space by one axis that measures compactness and one axis that measures the effective polytropic index. In the subregion that corresponds to NSs, i.e. for $C=\mathcal{O}(0.1)$ and $n \in[0.3,1]$, the isodensity profiles are approximately self-similar in the region inside the star that matters the most for the calculation of multipole moments, i.e. the eccentricity of isodensity contours remains invariant under a radial remapping. This is not the case for realistic noncompact stars which have large eccentricity variations in their interior. Thus, as compactness increases and one flows to the NS corner of phase space (see Fig. 3), an approximate symmetry emerges (isodensity contours become approximately self-similar) and this is responsible for the universality we have observed. As one approaches the region of phase space inhabited by $\mathrm{BHs}$, the universality becomes exact, as expressed by the no-hair theorems.

\section{FUTURE DIRECTIONS}

In this paper, we presented the relations among multipole moments for differentially-rotating stars in the Newtonian limit for the first time. The next task is to see how the universal relations change due to differential rotation in the relativistic case. For this, one could adopt the rotation law given in [80]. It would also be interesting to derive an analytic relation among multipole moments for differentiallyrotating stars and compare it with the relativistic results.

As explained in the Introduction, other types of universal relations exist, such as those among higher- $\ell$ tidal Love numbers [17] and NS oscillation modes [13,15,16]. It would be important to carry out a similar analysis as presented in this paper to understand why the universality 
holds among these observables. One can for example take a piecewise polytropic EOS and see which parameter affects the relations the most. One can also investigate which radial region affects the relations the most, and determine which region in the EOSs controls the universality.

Another interesting idea would be to study whether the $\bar{I}-\bar{Q}$ relations remain universal if one breaks the elliptical isodensity approximation. In this paper, we studied how the relation among multipole moments behaves as this approximation is relaxed. To repeat the analysis for the $\bar{I}-\bar{Q}$ relation, one would have to determine how the angular frequency-eccentricity relation is modified as one promotes the eccentricity to a radial function. Based on the results of this paper, we suspect the $\bar{I}-\bar{Q}$ relations will be similarly affected if one relaxes the elliptical isodensity approximation.

Finally, one could carry out a similar analysis as that performed in this paper but to study the origin of universality in theories other than GR, such as dynamical ChernSimons gravity [25,26], Eddington-inspired Born-Infeld gravity [40] and scalar-tensor theories [41]. It would be interesting to study how the variation in the NS eccentricity in the region that matters changes and see if the explanation found here in GR can also be applied to the multipole relations in such theories.

\section{ACKNOWLEDGMENTS}

We would like to thank Ben Owen, Jan Steinhoff, Terence Delsate and Katerina Chatziioannou for useful comments and suggestions. N. Y. acknowledges support from NSF Grant No. PHY-1114374 and the NSF CAREER Award No. PHY-1250636, as well as support provided by the National Aeronautics and Space Administration from
Grant No. NNX11AI49G, under sub-award 00001944. L.C.S. acknowledges that support for this work was provided by the National Aeronautics and Space Administration through Einstein Postdoctoral Fellowship Award No. PF2-130101 issued by the Chandra X-ray Observatory Center, which is operated by the Smithsonian Astrophysical Observatory for and on behalf of the National Aeronautics Space Administration under Contract No. NAS8-03060, and further acknowledges support from NSF Grant No. PHY-1068541. G. P. acknowledges financial support from the European Research Council under the European Union's Seventh Framework Programme (FP7/2007-2013)/ERC Grant Agreement No. 306425 "Challenging General Relativity." Some calculations used the computer algebra systems MAPLE, in combination with the GRTENSORII package [81].

Note added.-After we posted the preprint of this manuscript, Ref. [82] came out where the authors studied the I-Love-Q relations for proto-NSs with a nonbarotropic EOS. They found that at the early stage of the NS formation, when the entropy gradient inside the star is large, the relation differs from the one with barotropic EOSs by as much as $\sim 30 \%$. Moreover, they found that the eccentricity inside the star varies by $\sim 200 \%$ at this early stage of formation. This supports one of our claims, that the relation depends strongly on the stellar eccentricity variation. However, we note that Ref. [82] does not discuss how the universality itself changes with time for proto-NSs, as the authors only looked at a single nonbarotropic EOS. Namely, the reference does not provide any evidence on how the EOS universality itself depends on the eccentricity variation.
[1] J. M. Lattimer and M. Prakash, Astrophys. J. 550, 426 (2001).

[2] J. M. Lattimer and M. Prakash, Phys. Rep. 442, 109 (2007).

[3] J. M. Lattimer, Annu. Rev. Nucl. Part. Sci. 62, 485 (2012).

[4] F. Ozel, Rep. Prog. Phys. 76, 016901 (2013).

[5] A. W. Steiner, J. M. Lattimer, and E. F. Brown, Astrophys. J. 722, 33 (2010).

[6] J. M. Lattimer and A. W. Steiner, Astrophys. J. 784, 123 (2014).

[7] J. M. Lattimer and Y. Lim, Astrophys. J. 771, 51 (2013).

[8] J. M. Lattimer and A. W. Steiner, Eur. Phys. J. A50, 40 (2014).

[9] J. M. Lattimer and A. Yahil, Astrophys. J. 340, 426 (1989).

[10] M. Prakash, I. Bombaci, M. Prakash, P. J. Ellis, J. M. Lattimer, and R. Knorren, Phys. Rep. 280, 1 (1997).

[11] J. M. Lattimer and M. Prakash, Science 304, 536 (2004).
[12] P. Haensel, J. L. Zdunik, M. Bejger, and J. M. Lattimer, Astron. Astrophys. 502, 605 (2009).

[13] N. Andersson and K. D. Kokkotas, Mon. Not. R. Astron. Soc. 299, 1059 (1998).

[14] O. Benhar, V. Ferrari, and L. Gualtieri, Phys. Rev. D 70, 124015 (2004).

[15] L. Tsui and P. Leung, Mon. Not. R. Astron. Soc. 357, 1029 (2005)

[16] H. Lau, P. Leung, and L. Lin, Astrophys. J. 714, 1234 (2010).

[17] K. Yagi, Phys. Rev. D 89, 043011 (2014).

[18] K. Kiuchi, Y. Sekiguchi, M. Shibata, and K. Taniguchi, Phys. Rev. Lett. 104, 141101 (2010).

[19] K. Kyutoku, M. Shibata, and K. Taniguchi, Phys. Rev. D82, 044049 (2010); D84, 049902(E) (2011).

[20] K. Kyutoku, H. Okawa, M. Shibata, and K. Taniguchi, Phys. Rev. D 84, 064018 (2011). 
[21] A. Bauswein and H.-T. Janka, Phys. Rev. Lett. 108, 011101 (2012).

[22] S. Bernuzzi, A. Nagar, S. Balmelli, T. Dietrich, and M. Ujevic, Phys. Rev. Lett. 112, 201101 (2014).

[23] K. Takami, L. Rezzolla, and L. Baiotti, Phys. Rev. Lett. 113, 091104 (2014).

[24] M. AlGendy and S. M. Morsink, Astrophys. J. 791, 78 (2014).

[25] K. Yagi and N. Yunes, Science 341, 365 (2013).

[26] K. Yagi and N. Yunes, Phys. Rev. D 88, 023009 (2013).

[27] D. G. Ravenhall and C. J. Pethick, Astrophys. J. 424, 846 (1994).

[28] M. Bejger and P. Haensel, Astron. Astrophys. 396, 917 (2002).

[29] J. M. Lattimer and B. F. Schutz, Astrophys. J. 629, 979 (2005).

[30] M. Urbanec, J. C. Miller, and Z. Stuchlík, Mon. Not. R. Astron. Soc. 433, 1903 (2013).

[31] A. Maselli, V. Cardoso, V. Ferrari, L. Gualtieri, and P. Pani, Phys. Rev. D 88, 023007 (2013).

[32] B. Haskell, R. Ciolfi, F. Pannarale, and L. Rezzolla, Mon. Not. R. Astron. Soc. 438, L71 (2014).

[33] G. Pappas and T. A. Apostolatos, Phys. Rev. Lett. 112, 121101 (2014).

[34] S. Chakrabarti, T. Delsate, N. Gurlebeck, and J. Steinhoff, Phys. Rev. Lett. 112, 201102 (2014).

[35] K. Yagi, K. Kyutoku, G. Pappas, N. Yunes, and T. A. Apostolatos, Phys. Rev. D 89, 124013 (2014).

[36] L. C. Stein, K. Yagi, and N. Yunes, Astrophys. J. 788, 15 (2014).

[37] K. Chatziioannou, K. Yagi, and N. Yunes, arXiv:1406.7135.

[38] R. Jackiw and S. Y. Pi, Phys. Rev. D 68, 104012 (2003).

[39] S. Alexander and N. Yunes, Phys. Rep. 480, 1 (2009).

[40] Y. H. Sham, L. M. Lin, and P. Leung, Astrophys. J. 781, 66 (2014).

[41] P. Pani and E. Berti, Phys. Rev. D 90, 024025 (2014).

[42] K. C. Gendreau, Z. Arzoumanian, and T. Okajima, in Proceedings of the Society of Photo-Optical Instrumentation Engineers (SPIE) Conference Series, Vol. 8443 (SPIE, Bellingham, WA, 2012).

[43] P. S. Ray, M. Feroci, J. den Herder, E. Bozzo, and L. Stella (LOFT Collaboration), in American Astronomical Society Meeting Abstracts, Vol. 219 (AAS, Washington, DC, 2012) p. 249.06 .

[44] M. Feroci, J. W. den Herder, E. Bozzo, D. Barret, S. Brandt, M. Hernanz, M. van der Klis, M. Pohl, A. Santangelo, L. Stella et al., in Proceedings of the Society of Photo-Optical Instrumentation Engineers (SPIE) Conference Series, Vol. 8443 (SPIE, Bellingham, WA, 2012).

[45] D. Psaltis, F. Ozel, and D. Chakrabarty, Astrophys. J. 787, 136 (2014).

[46] M. Baubock, E. Berti, D. Psaltis, and F. Ozel, Astrophys. J. 777, 68 (2013).

[47] C. W. Misner, K. Thorne, and J. A. Wheeler, Gravitation (Freeman, San Francisco, 1973).

[48] D. Robinson, Phys. Rev. Lett. 34, 905 (1975).

[49] W. Israel, Phys. Rev. 164, 1776 (1967).

[50] W. Israel, Commun. Math. Phys. 8, 245 (1968).

[51] S. Hawking, Phys. Rev. Lett. 26, 1344 (1971).

[52] S. W. Hawking, Commun. Math. Phys. 25, 152 (1972).
[53] B. Carter, Phys. Rev. Lett. 26, 331 (1971).

[54] D. Lai, F. A. Rasio, and S. L. Shapiro, Astrophys. J. Suppl. Ser. 88, 205 (1993).

[55] J. B. Hartle, Astrophys. J. 150, 1005 (1967).

[56] J. B. Hartle and K.S. Thorne, Astrophys. J. 153, 807 (1968).

[57] J. S. Read, B. D. Lackey, B. J. Owen, and J. L. Friedman, Phys. Rev. D 79, 124032 (2009).

[58] F. Douchin and P. Haensel, Astron. Astrophys. 380, 151 (2001).

[59] N. Stergioulas and J. L. Friedman, Astrophys. J. 444, 306 (1995).

[60] M. Rieutord and F. Espinosa Lara, in Lecture Notes in Physics, Berlin Springer Verlag, Vol. 865, edited by M. Goupil, K. Belkacem, C. Neiner, F. Lignières, and J. J. Green (Springer-Verlag, Berling, 2013), p. 49.

[61] F. Espinosa Lara and M. Rieutord, Astron. Astrophys. 552, A35 (2013).

[62] A. Vaezi, M. Mashkoori, and M. Hosseini, Phys. Rev. B 85, 195126 (2012).

[63] S. L. Shapiro and S. A. Teukolsky, Black Holes, White Dwarfs and Neutron Stars: The Physics of Compact Objects (Wiley, New York, 1986), p. 672.

[64] J. S. Read, C. Markakis, M. Shibata, K. Uryū, J. Creighton, and J. Friedman, Phys. Rev. D 79, 124033 (2009).

[65] A. Akmal, V. Pandharipande, and D. Ravenhall, Phys. Rev. C 58, 1804 (1998).

[66] J. M. Lattimer and F. Douglas Swesty, Nucl. Phys. A535, 331 (1991).

[67] H. Shen, H. Toki, K. Oyamatsu, and K. Sumiyoshi, Nucl. Phys. A637, 435 (1998).

[68] H. Shen, H. Toki, K. Oyamatsu, and K. Sumiyoshi, Prog. Theor. Phys. 100, 1013 (1998).

[69] M. Prakash, J. Cooke, and J. Lattimer, Phys. Rev. D 52, 661 (1995).

[70] V. R. Pandharipande and R. A. Smith, Nucl. Phys. A237, 507 (1975).

[71] R. F. Tooper, Astrophys. J. 140, 434 (1964).

[72] F. D. Ryan, Phys. Rev. D55, 6081 (1997).

[73] J.-L. Tassoul, Theory of Rotating Stars, Princeton Series in Astrophysics (Princeton University, Princteon, NJ, 1978).

[74] R. A. Brooker and T. W. Olle, Mon. Not. R. Astron. Soc. 115, 101 (1955).

[75] T. Mora and C. M. Will, Phys. Rev. D 69, 104021 (2004).

[76] E. M. Butterworth, Astrophys. J. 204, 561 (1976).

[77] C. A. Iglesias and F. J. Rogers, Astrophys. J. 464, 943 (1996).

[78] D. D. Doneva, S. S. Yazadjiev, N. Stergioulas, and K. D. Kokkotas, Astrophys. J. 781, L6 (2014).

[79] M. Galassi and B. Gough, GNU Scientific Library: Reference Manual, GNU Manual (Network Theory Limited, United Kingdom, 2009).

[80] H. Komatsu, Y. Eriguchi, and I. Hachisu, Mon. Not. R. Astron. Soc. 237, 355 (1989).

[81] GRTensorII is a package that runs within Maple but is distinct from packages distributed with Maple. It is distributed freely at http://grtensor.org.

[82] G. Martinon, A. Maselli, L. Gualtieri, and V. Ferrari, arXiv:1406.7661. 